\title{
Functional reassembly of membrane proteins in planar lipid bilayers
}

\author{
M. MONTAL, $†$ A. DARSZON† AND H. SCHINDLER
}

†University of California, San Diego, La folla, California 92093, USA.

†Centro de Investigacion del Instituto Politecnico Nacional, Mexico, 14, D.F. Mexico. §Biozentrum, University of Basel, Basel, Switzerland

(I) GeneraL

(A) Introduction 2

(B) Methodological requirements for a successful reconstitution 2

(i) Reference data 2

(ii) Parameters of reconstitution 7

(iii) Adjustment of variables 8

(iv) Problems in reconstitution - identification of artifacts 9

(C) Approaches for the reassembly of membrane proteins in planar bilayers 10

(i) Monolayer self-assembly from protein-lipid complexes in organic solvents 10

(ii) Monolayer self-assembly from (protein-)lipid vesicle suspensions 18

(iii) Fusion of preformed (protein-)lipid vesicles with preformed planar bilayers 19

(iv) Incorporation of detergent-solubilized membrane extracts into preformed planar bilayers 22

(II) CHANNELS

(A) Rhodopsin 22

(i) Background 22

(ii) Vesicles 25

(iii) Monolayers and planar bilayers 25

(B) Acetylcholine receptor 30

(i) Background 30 
(ii) Vesicles 33

(iii) Planar bilayers 36

(C) Porin 40

(i) Background 40

(ii) Vesicles 40

(iii) Planar bilayers $4^{\circ}$

(D) Proton channel of the proton-translocating ATPase 46

(i) Background 46

(ii) Vesicles 46

(iii) Planar bilayers

(III) PUMPS

(A) Reaction centers from photosynthetic bacteria 48

(i) Background 48

(ii) Large vesicles 50

(iii) Planar bilayers $5^{1}$

(B) Cytochrome c oxidase 55

(i) Background $\mathbf{5 5}$

(ii) Vesicles $5^{6}$

(iii) Planar bilayers $5^{8}$

(C) Bacteriorhodopsin 59

(i) Background 59

(ii) Vesicles and interfacial films 60

(iii) Planar bilayers 63

(IV) Perspectives in membrane reconstitution

(V) REFERENCES

(I) GENERAL

(A) Introduction

Recent progress in membrane biology has brought us to a stage where it is possible to associate complex biological processes to identifiable membrane proteins. Technical advances in the biochemical characterization and purification of membrane proteins have contributed a wealth of structural information. The reconstitution approach has proved to be valuable in our efforts to understand the molecular 
mechanisms of membrane transport and energy transduction. This approach allows, in principle, the dissection of a complex phenomenon into its components with the subsequent gain of experimental control over parameters that are otherwise inaccessible or highly variable in the natural membrane. In addition, it establishes general principles for the reassembly of biologically active membranes providing insight into the interplay between the membrane environment and the functional unit.

In 1962, Mueller et al. introduced the planar lipid membrane as a model system to study the molecular basis of transport in biological membranes. This system, the so called black lipid film, has been extremely useful in establishing possible modes of transport across membranes, such as through transmembrane channels or via mobile carriers. This success was accomplished through the advent of polypeptide and polyene antibiotics. However, progress in the reassembly of planar bilayers from lipids and integral membrane proteins in a functional state has lagged behind. In the last few years basic conceptual and methodological advances in the reassembly of functional membrane proteins in planar lipid bilayers have opened new perspectives and challenges. In this review, we attempt to describe the salient features of these developments and their applications to selected systems. Throughout this review we distinguish between membrane reconstitution and membrane reassembly by assigning a functional connotation to the first.

The rationale for using planar bilayers in membrane reconstitution studies resides in the adequacy of the experimental system for the sensitive and detailed characterization of electrical events occurring in membranes. The most salient application of planar membrane reconstitution is; therefore, to membrane phenomena associated to the translocation of charges across the lipid bilayer, namely channels and pumps. For the sake of clarity, the following introductory discussion is elaborated using an ion-channel as a prototype entity; one should bear in mind that the concepts are not restricted to channels.

It is not our intention to propose a particular reconstitution scheme but to examine some basic methodological requirements which may help to visualize the progress so far attained as well as to appreciate the unresolved problems and to identify ways to further develop reconstitution strategies and assays. 


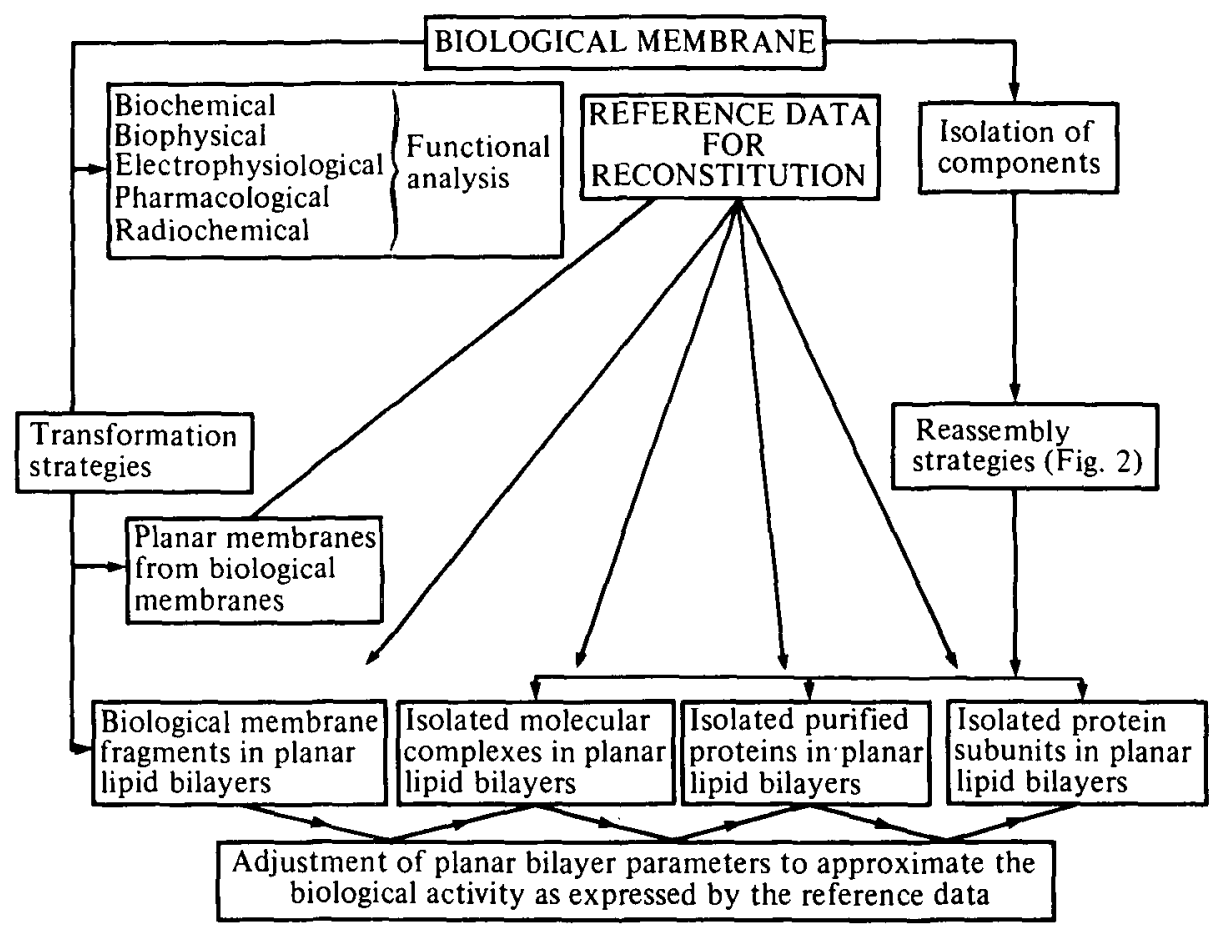

Fig. I.

(B) Methodological requirements for a successful reconstitution

(i) Reference data

Reconstitution involves certain capabilities represented as a flow diagram in Fig. I. It shows, in addition to the isolation of components and their reincorporation into planar lipid membranes, other important pathways of reconstitution work. The diagram illustrates the requirement of reference data acquired through a functional analysis of the transport system of interest in order to evaluate the observations on reassembled membranes. An alternative source of reference data is presented. This involves data obtained in vitro by the transformation of native membrane vesicles into planar membranes. Beyond protein reincorporation into a membrane environment, reconstitution is concerned with the improvement of observed data toward the reference data. To achieve this, the incorporation of a membrane protein may not suffice since the participation of other membrane parameters can play an important role in the function. Therefore, a detailed adjustment of the 
membrane environment including composition, organization and state of each monolayer of the planar bilayer, as well as the control of the aqueous phases is probably required. The last aspect in the figure indicates how reconstitution attempts and the adjustment of the required membrane parameters may be based on a systematic approach. This strategy emerges from the possibility of reconstitution from different starting conditions: from unperturbed native membrane fragments, crude membrane extracts, single isolated and purified membrane components or even only their subunits. Reconstitution at these different degrees or preservation of the original membrane conditions, from higher to lower degrees, allows the sequential identification of the requirements for functional reassembly. This approach substitutes the need to resolve all these requirements simultaneously when reconstitution is restricted to the level of single components. This strategy aims to minimize the effort to resolve the investigated system function into its constituent parts and the environmental conditions.

An inherent feature of the reconstitution approach is the existence from the outset of a mismatch between observed and reference data. The strategies taken to accomplish further adjustments of observed to reference data contain the desired information about specific requirements to restore the original channel function. The identification, realization and analysis of the appropriate actions taken to successfully achieve a functional reconstitution constitute both the virtue as well as the difficulty of the approach.

From this central consideration, the demands on a reconstitution assay are inferred; these will be expressed as three methodological conditions, in addition, to the requirement of detailed reference data. The channel properties (such as single-channel conductance, channel conductance states, transition rates between channel states, ionic strength dependence, ion selectivity, current-voltage characteristics of the channel and of an ensemble of channels, interactions between channels) will be referred to as functional variables. In order to exploit the specific virtues of planar lipid bilayers in reconstitution of channel function it is necessary to count with in vivo data about the channel properties which are expected to or found to vary in the reassembled membrane including the information derived from single-channel recordings. This apparent paradox is not due to a too exacting view of reconstitution but is a direct consequence of the consideration that the channel properties may not be uniquely defined by single values during 


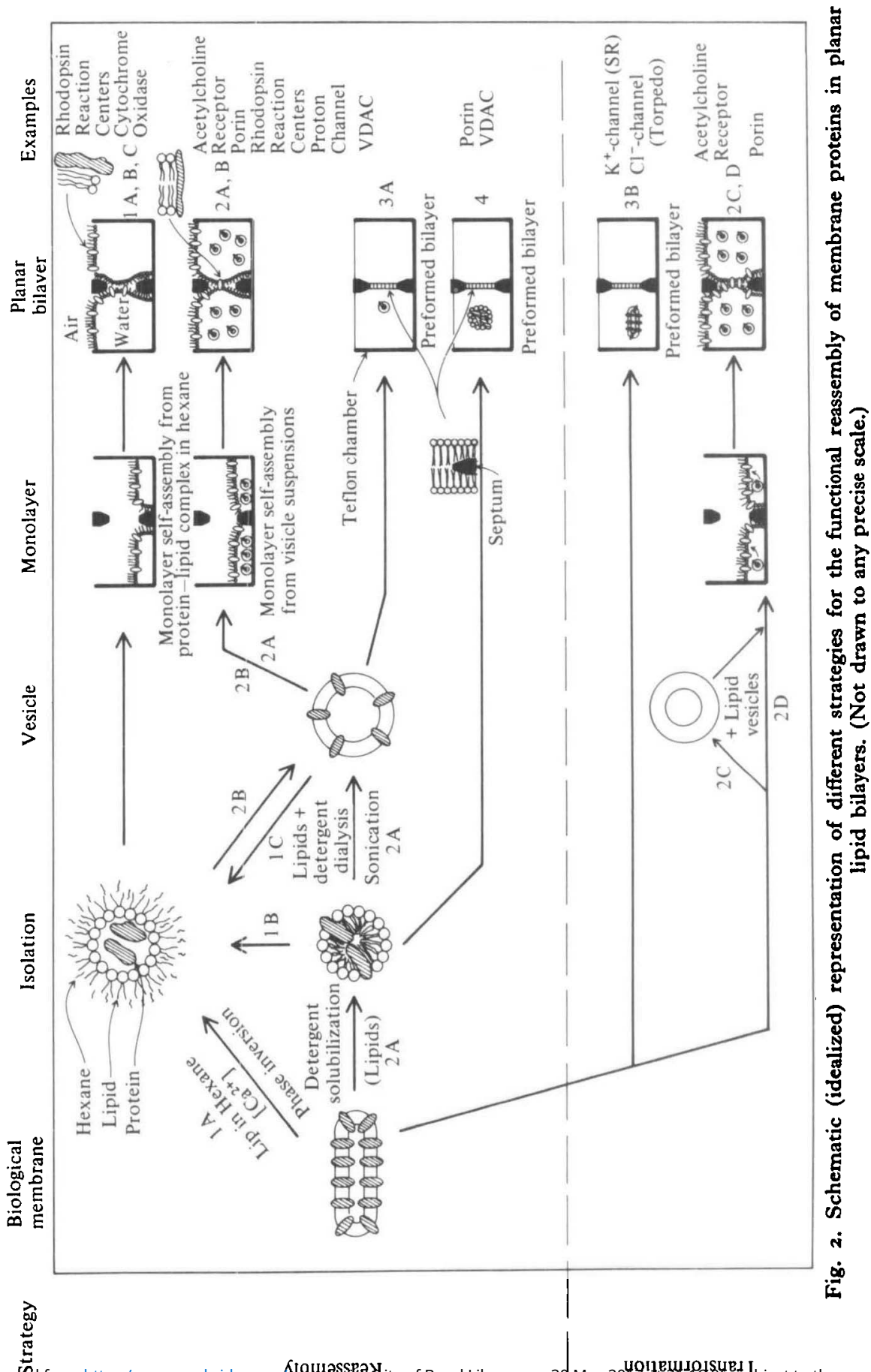


reconstitution attempts. It is precisely in this variation of the channel characteristics that resides the potential of reconstitution in order to identify molecular and membrane conditions required for the physiological function.

The characteristics of the in vivo channel derived from the biochemical, biophysical or pharamacological functional analysis are needed. The prominent tool, however, is electrophysiology. Unfortunately there are only few membrane channels which have been characterized in such detail using noise analysis and the recently developed patch clamp technique (Neher \& Sakmann, 1976; Neher \& Steinbach, 1978). The majority of electrical membrane phenomena are, due to spatial constraints, not accessible to current electrophysiological characterization. One alternative approach to study the electrical properties of natural membranes in vitro would be to form a planar membrane from isolated membranes. Evidently, this would be at the expense of removing the membrane from its in vivo environment. Recently, such a method was developed (strategy 2 C, D in Fig. 2) and successfully applied to Escherichia coli outer membranes (see page 50, Schindler \& Rosenbusch, 1980), vesicular membrane fragments from electric organs of the Torpedo fish (see page 45, Schindler, \& Quast, 1980). However, the pressing need of finding more direct ways to form planar membranes from biological membranes with preservation of native organization of membrane constituents prevails. Fusion of natural membranes with preformed planar lipid bilayers (strategy 3 in Fig. 2) may be considered but the overall composition and state of the resulting bilayer is different from that of the original membrane even if one assumes that the fusion process, which is currently poorly understood, results in a direct intercalation of membrane fragments into the host lipid membrane. This approach has its own virtues as outlined below.

(ii) Parameters of reconstitution

In general, the functional variables of a channel may not be uniquely determined by the intrinsic properties of the particular molecular entity which constitutes the channel but may depend on properties of the membrane - or the aqueous environment. How membrane parameters influence transport functions is a question of general interest and widely unresolved. The reconstitution of a channel function as an approach to answer this question requires the ability to measure and change membrane parameters. Parameters, which are most likely to 
influence the functional variables are related to membrane composition and energy. For the moment, parameters of the aqueous environment are disregarded.

The primary parameters, are the concentrations of all components in the reassembled planar lipid bilayer including the composition of its two monolayers and also parameters which define the membrane energy state (temperature, surface free energy or lateral pressure). Strategies $I$ and 2 in Fig. 2 allow the assembly of planar lipid bilayers with known and adjustable composition in each half of the membrane.

As the observables in planar lipid bilayer studies are the electrical data, we are led to ask if and to what extent the electrical activity of a membrane channel contains information about organization, reorganization and interaction of the channel constituents and other membrane components. This question may be approached as follows. (I) Detailed analysis of concentration dependences, voltage dependences of the channel activity or amplitude and time correlation of the signals. The electrical signals contain primarily information about molecular processes in the immediate molecular periphery of the channel, and have been used successfully to elucidate mechanisms of channel formation by peptide antibiotics. Dependences of the electrical signals on the concentration of the membrane components may reveal association with, to or of ionophoric units. (2) Use of defined initial lateral distribution of components in the planar membrane to study the role of constituent association or dissociation in channel function. Strategies $\mathrm{I}$ and 2 in Fig. 2 can be used to impose upon the membrane a variety of different random and nonrandom initial distributions, laterally and in each monolayer of the bilayer. Information is derived from the rise or change of channel conductance in time which can be assigned to particular components and, for example, to their association via lateral diffusion.

\section{(iii) Adjustment of variables}

In the two preceding sections the prerequisites for reconstitution, the demand on a reconstitution assay that allows adjustment of primary membrane parameters to measured parameters and the need for reference data to assign a certain state of the reassembled membrane to the physiological system were considered.

We now turn to the question of how to proceed towards reconstitution. This endeavour has opened with the development of techniques to form planar lipid bilayers containing biological membrane materials at 
different degrees of structural and therefore functional preservation (see Fig. 2): Planar lipid bilayers formed from only natural membrane materials (transformation strategies $2 \mathrm{C}, 2 \mathrm{D}, 2 \mathrm{E}$ ), from natural membrane fragments and lipids (strategies $\mathrm{I}$ and 2 ) and from lipids and isolated membrane constituents at various degrees of isolation and purification down to subunits of membrane proteins (reassembly strategies 1, 2, $3 \mathrm{~A}, 4$ ). These reassembled membranes differ in their level of resolution. At the lowest level of resolution the restoration of a functional membrane state requires fewer parameters to adjust but, consequently, less information can be derived. It is now possible to start reconstitution at the lowest level of resolution and to proceed stepwise towards higher levels. This is illustrated in Fig. I. One principal advantage of this procedure is that the number of problems or unmatched membrane parameters at any level of resolution and reconstitution can be held low due to the knowledge obtained at the lower levels. Fusion (strategy 3 ) is an alternate procedure and its application to the sarcoplasmic reticulum membrane will be described.

\section{(iv) Problems in reconstitution - identification of artifacts}

Reconstitution necessarily involves situations where the functional variables in the reassembled membranes do not match the reference data. This may be due to requirements on membrane parameters which have not been met. Alternatively or in addition this discrepancy may arise from structural modifications of the membrane constituents during isolation, purification or membrane assembly. The discrimination between these two causes is extremely difficult. Their effects on functional variables offer no basis for their discrimination, neither do procedure modification unless functional reconstitution is achieved. If clear identification of artifacts were hindered the condition remains on how to collect indications for the presence of artifacts so that one might invest further efforts in the right direction. For this purpose it is most advantageous to use different levels of resolution and reconstitution. If at the lowest level agreement is found between observed and reference data one may trust the reincorporation procedure also at higher levels. In case of disagreement, the cause may likewise reside in the membrane formation which should be improved before reconstitution attempts are carried to higher levels of resolution. At higher levels the problem of artifacts remains but somewhat restricted to isolation or purification. 
Also, with few parameters to adjust at any particular level, reconstitution is not only considerably facilitated but artifacts may be more clearly identified. Thus, the problem of artifacts is reduced but it is only solved by the actual functional reconstitution.

\section{(C) Approaches for the reassembly of membrane proteins in planar bilayers}

There are two conceptual modalities for the reassembly of membrane proteins into planar lipid bilayers, namely the sequential and the simultaneous.

(i) Sequential reassembly. This modality has been most widely used in reconstitution studies. In essence, it consists of the adsorption of protein on preformed structures with the eventual incorporation into the membrane. The preformed planar membrane can be assembled either as black lipid films (solvent-containing) (Mueller et al. 1962) or as bilayers formed by adjoining the hydrocarbon chains of two lipid monolayers (Montal \& Mueller, 1972). To incorporate the protein into the preformed structure two pathways have been used: the protein or a membrane extract is introduced into the aqueous phase either as a detergent extract (strategy 4, Fig. 2) or as a vesicle suspension (strategy ${ }_{3} \mathrm{~A}$ or $3 \mathrm{~B}$, Fig. 2). The latter approach has been elaborated in more detail and is referred to as fusion of vesicles with planar bilayers.

(ii) Simultaneous assembly. This alternative view considers the concerted assembly of lipid and protein components. It is evident from the strategies outlined in Fig. 2 that an intermediate step in planar bilayer formation (Montal \& Mueller, 1972) is the generation of monolayers at the air-water interface. Simultaneous assembly implies incorporation of lipid and protein into the monolayer. There are two main pathways to achieve the self-assembly of a lipid-protein monolayer: either from lipid-protein complexes in organic solvents (strategy I, Fig. 2) or from vesicle suspensions (strategy 2, Fig. 2). We now turn to describe in more detail the different strategies illustrated in Fig. 2.

(i) Monolayer self-assembly from protein-lipid complexes in organic solvents (strategies I A-C, Fig. 2)

A manoeuvre to reassemble a membrane protein-containing planar lipid bilayer involves the transfer of protein-lipid complexes (PLC) $†$ into

† Abbreviations used: AChR, Acetylcholine receptor; ATP, Adenosine triphosphate; DCCD, dicyclohexylcarbodiimide; EPR, electron paramagnetic resonance; FCCP, flurocarbonylcyanidephenylhydrazone; GMP, guanosine monophosphate; 
organic solvents (Montal, 1974). Although the original motivation to develop the necessary techniques for the extraction of PLCs into organic solvents was to use them in planar bilayer formation, they are now used as building blocks of several other model membranes (as shown in Fig. 2): they spread into monolayers at air-water interfaces (Montal, 1974; Trissl, Darszon \& Montal, 1977), large proteincontaining vesicles can be formed by either sonication of PLCs in the solvent with aqueous buffer followed by solvent evaporation under reduced pressure (Darszon et al. 1979c) or by evaporation of the solvent and slow rehydration of the residue (Darszon et al. 1980).

Organic solvents have been extensively used to extract small hydrophobic polypeptides from diverse membrane systems (Zahler \& Niggli, 1977; Lees et al. 1979). Proteolipids from sarcoplasmic reticulum, the mitochondrial DCCD-binding protein (Sigrist, Sigrist-Nelson \& Gitler, 1977) and an active DCCD-binding protein from chloroplasts (Nelson et al. 1977) were extracted in single phase system using $n$ butanol.

The possibility of developing a more general procedure to transfer membrane proteins in active form into organic solvents emerged from several considerations. Membrane proteins usually have hydrophilic and hydrophobic regions. It can be assumed that the hydrophobic parts will be at least partially soluble in apolar solvents while the hydrophilic regions will not. A possible strategy to induce the partition of the protein into the apolar environment would be to neutralize their charge by forming ion pairs (Gitler \& Montal, 1972a, b). For instance, it appears that the solubility of some proteolipids in organic solvents involve preferential interactions between the apoprotein and acidic phospholipids (Lees et al. 1979) probably forming ion pairs (Gitler \& Montal, $1972 a$ ). The interaction energy between two charges is inversely related to the dielectric constant of the medium, therefore electrostatic interactions would be favoured as the polarity of the medium decreases (Salem, I962). Ions (Gemant, 1962) and ion pairs are known to exist in hydrocarbon solvents. Consequently, ion pairs between proteins and lipid probably exist in the hydrophobic regions of membranes (Montal, 1976).

Initially it was shown that stoichiometric complexes composed of neutral and acidic lipids and cytochrome $c$ could be extracted into GTP, guanosine triphosphate; PLC, protein-lipid complex; RLC, rhodopsin-lipid complex; RC, reaction centers; SR, sarcoplasmic reticulum; VDAC, voltage-dependent anion channel. 
isooctane (Das \& Crane, 1964). The partition of these complexes into apolar solvents was enhanced by the charge neutralization (ion pair formation) between the positive charges of cytochrome $c$ and acidic phospholipids. Using a modification of the protocol established by Das \& Crane (1964) it was found that the partition of cytochrome $c$-lipid complexes into decane was enhanced either by lowering the $\mathrm{pH}$ or adding cations, $\mathrm{Ca}^{2+}$ being the most effective ion (Gitler \& Montal, I $972 a, b)$. The formation of black lipid membranes from these extracts suggested the potential use of this strategy for the reconstitution of complex membrane functions (Gitler \& Montal, 1972 $a, b$; Montal, 1974).

Following this approach, the principles developed (Das \& Crane, 1964; Gitler \& Montal, 1972a,b) were used to attempt the extraction into organic solvents of detergent-solubilized and partially purified membrane proteins in their active form (Montal, 1974; Montal \& Korenbrot, 1973). Cytochrome oxidase was extracted into hexane as a protein-lipid complex with high yield $(90 \%)$ and its partition was dependent on the presence of $\mathrm{Ca}^{2+}$. Its enzymatic activity was retained after solvent evaporation, both in liposomes made by rehydrating the protein-lipid residue and in monolayers at the air water interface (Montal, r974) (see Section III, B (ii)).

Using rhodopsin, the visual pigment from vertebrate photoreceptor cells as a model membrane protein, a general strategy was devised for the extraction of PLCs into organic solvents to be used as starting material for reconstitution in bilayer membranes (Darszon et al. 1978). The strategy was based on the systematic evaluation of the variables which determined an optimum and reproducible performance (Montal, 1974; Montal \& Korenbrot, I973; Darszon, Montal \& Philipp, 1977a; Darszon et al. 1978). The approach involved describing the composition of the PLCs in the solvent and an analysis of the conditions which allowed the preservation of the native characteristics of the protein. Two distinct but complementary procedures were developed to transfer protein-lipid complexes from aqueous into non-aqueous phases (Darszon et al. I978). The first allows the solubilization of the protein in detergent and its subsequent purification and delipidation if needed; after partial detergent removal and recombination with the desired phospholipids in the aqueous phase, the lipoprotein vesicles are extracted with organic solvents. The major advantage of this procedure is to yield extracts derived from a purified membrane protein where the experimenter can also control the lipid composition (Darszon et al. 
I979a). This is the procedure of choice in reconstitution studies where the membrane system contains several proteins, since solubilization and purification of the protein of interest are desirable. The main limitation of this version is the presence of residual detergent. In contrast, the second method involves the direct transfer of the protein as a PLC from the biological membrane into a non-aqueous phase: an aqueous pellet of purified membranes resuspended in an organic solvent supplemented with phospholipids is sonicated, cations are mixed and the two phases separated by centrifugation. These extracts have never been in contact with detergent, and therefore are instrumental in defining the role of detergents in the phenomena studied. In this respect the detergent-containing preparation is valuable in dissecting the involvement of one or more components in the behaviour of the reconstituted membranes. Thus the two procedures complement each other. The choice of the solvent used in the PLC extraction is determined by the preservation of biological activity and optimum yield. In general hexane and other alkanes are suitable for all the proteins that have been tested thus far. It was found that a second extraction with pure ethyl ether could be performed on the emulsified aqueous phase left after the first extraction into hexane. This second ether extract has the advantage of having a lower lipid to protein ratio, therefore by adding lipid into the ether one can control this ratio, from 200 which approaches that existent in the retinal rod disk membranes ( $\cong 75$ ) (Darszon et al. 1980 ) to several thousands.

The results indicate that rhodopsin can be transferred into hexane and ether as a PLC in good yields using the procedures mentioned. Rhodopsin from this extract retains the dark and bleached spectral characteristics it displays in cell membranes (Darszon et al. 1978; Darszon, Strasser \& Montal, $1979 b$ ). Furthermore, the ability of opsin to recombine with either I I-cis or 9-cis retinal to form rhodopsin or isorhodopsin respectively, a very sensitive assay of the native state of the protein (Hubbard \& Wald, I952), was preserved in the rehydrated opsin-lipid residue left after solvent evaporation from the extracts (Darszon et al. 1978; Darszon et al. 1979b).

The action mechanism of rhodopsin is not known, therefore, the evaluation of the functionality of RLCs for their use in reconstitution studies had to be based on a comparison of other known characteristics of the protein in its natural environment. The ultimate test for the presence of spectrally native rhodopsin in reconstituted planar bilayers would be to record the absorption spectra of the bilayer itself. At 
present this is technically difficult. To circumvent this problem, a system was developed to study the material from which the bilayers are formed, under similar conditions in which the bilayer is studied (Darszon et al. $1979 b$ ). Rhodopsin was transferred directly from purified rod outer segments as a PLC into hexane or ether. Thereafter, the ether extract was layered upon a filter paper disk used as a rigid, porous matrix, and then hydrated as to stimulate the process of spreading the extract at air-water interfaces. The difference absorbance spectra of rhodopsinlipid complexes in hexane or ether between dark and irradiated samples show $\lambda_{\max }$ at $499 \pm \mathrm{I} \mathrm{nm}$ and isosbestic points around $4 \mathrm{r} 5 \pm 4 \mathrm{~nm}$. Bleached rhodopsin in hexane, but not in ether, when supplemented with 9-cis retinal, regenerated isorhodopsin with a yield of $\cong 90 \%$. In the filter paper rhodopsin showed absorption spectra in the dark and after bleacing similar to those recorded in ROS membranes; on addition of I I-cis retinal to the filter paper, regeneration of rhodopsin to $\cong 95 \%$ was obtained (Darszon et al. 1979b).

In a more detailed analysis of the photochemical functionality of the rhodopsin-lipid complexes in the filter paper using low-temperature spectroscopy to study the bleaching sequence, the following intermediates were observed : bathorhodopsin, lumirhodopsin, metarhodopsin I, metarhodopsin II and metarhodopsin III (Darszon et al. 1979b).

The kinetics of the metarhodopsin I-II transition, another important evaluation of the photochemical functionality of rhodopsin in the extracted form (Hagins, 1972; Ostroy, 1977), were also studied in the filter paper and in the solvents (Darszon et al. 1979b). The temperature and $\mathrm{pH}$ dependences of this transition in the rhodopsin-lipid complexes studied in the filter paper disk were comparable to the values reported for rhodopsin in rod disk membranes (Von Sengbusch \& Stieve, 1971; Applebury et al. 1974; Hoffman et al. 1978). In hexane the transition occurred in a similar manner. In contrast, the process was drastically different in ether, the formation and decay of metarhodopsin I appeared to proceed very fast (less than $100 \mathrm{~ms}$ at $0^{\circ} \mathrm{C}$ ) and was followed by a slower recovery of absorbance at the $\lambda_{\max }$ of metarhodopsin I. The marked dependence of the reaction kinetics on the nature of the solvent establishes that the protein is sensitive to the environment. This observation taken together with fact that rhodopsin is chemically regenerable in hexane but not in ether (Darszon et al. 1978) suggests that bleached rhodopsin is susceptible to denaturation in ether but not in hexane. 
Recently, using a combination of the two described procedures, it has been possible to transfer into hexane affinity-purified and delipidated rhodopsin, as a complex with a single lipid species which, in addition, is nonoxidizable (diphytanoylphosphatidylcholine). Rhodopsin from these complexes is photochemically active, as assayed by its dark and bleached spectra and its ability to regenerate isorhodopsin(Darszon et al. 1979a).

In general the results indicate the spectral integrity of the rhodopsinlipid complexes in hexane. Although the RLCs in ether appear to go through a different bleaching pathway and are not regenerable, when the solvent is evaporated in the dark and the residue studied in a filter paper, the rhodopsin spectral features are fully expressed, displaying a bleaching sequence, metarhodopsin I-II kinetics and regeneration capacity, similar to that of rhodopsin in rod outer segments. All of these criteria establish the competence of the rhodopsin-lipid complexes as an appropriate starting point for reconstitution in bilayer membranes (Darszon et al. 1979b).

The use of the methods just described to transfer biologically active proteins from membranes into apolar solvents as protein-lipid complexes, has already proved to be successful in other membrane systems. Examples of this are: cytochrome oxidase (Montal, 1974; Chien \& Mueller, 1976), bacteriorhodopsin (Hwang, Korenbrot \& Stoeckenius, r977a, b), a mitochondrial anion channel (Schein, Colombini \& Finkelstein, 1976) invertebrate squid rhodopsin (Vandenberg, unpublished results; Darszon et al. 1979c) and reaction centers from photosynthetic bacteria (Schönfeld, Montal \& Feher, 1979; KendalTobias \& Crofts, 1979; Packham et al. 1980).

The purified photosynthetic reaction center protein (RC) from photosynthetic bacteria has been transferred as a PLC into organic solvents using the approach described and the complexes thoroughly characterized (Schönfeld, Montal \& Feher, 1980). The extraction protocol included magnesium, but it could be replaced by calcium, and hexane, which could be replaced by other alkanes but not by more polar organic solvents.

In the visible and infrared regions the absorbance spectrum of the hexane extract, was similar to that of RCs in aqueous detergent solution. The light-induced absorbance changes were only about $5 \%$ of the expected values; however, the photoactivity could be almost fully restored by adding exogenous ubiquinone Qro. This indicated that the quinones were extracted by the organic solvent resulting initially in loss 
of activity. EPR spectroscopy was also used to evaluate the reaction center-lipid complex. Active reaction centers display two light-induced EPR signals. A narrow one at $g=2.0026$, associated with the oxidized primary electron donor $D$, and a broad signal at $g=\mathbf{I} \cdot 8$ associated with the reduced primary acceptor $\mathrm{A}$, a ferroquinone complex (Feher \& Okamura, 1978). The light-induced EPR absorbance changes and the low-temperature kinetics of back reactions in the dark were found to be similar to those determined for reaction centers in the chromatophore membrane or when purified. In terms of the composition of the reaction center-lipid complexes their molar ratios of phospholipid/RC, detergent/RC, $\mathrm{H}_{2} \mathrm{O} /$ phospholipid and $\mathrm{RC} / \mathrm{Mg}^{2+}$ were $4000-6000,400,40$, and $\cong 2$, respectively. The presence of water in the complexes had been revealed previously in the case of rhodopsin-lipid complexes by the fact that rhodopsin bleaches in the organic extracts since it is known that the metarhodopsin I-II transition does not occur in the dry state (Wald, Durrell \& St George, 1950; Ostroy, 1977; Darszon et al. I979b).

These results showed that reaction centres can be extracted into organic solvents as protein-lipid complexes while preserving photochemical activity. Furthermore, the reaction center-lipid complex reacted with exogenous electron carriers dissolved in the bulk hexane phase, thus performing biochemical reactions in an organic solvent. Their photochemical activity was also retained after solvent removal and rehydration, thus fulfilling the requirements for the successful reconstitution of the protein in functional bilayers. And indeed such a preparation has been used to form functional planar lipid bilayers (Schönfeld et al. 1979) and large vesicles (Darszon et al. Ig80) (see Section III, A).

The approach of directly transferring proteins from membranes to solvents is applicable, in principle, to any membrane system. The procedure is particularly suitable, though not exclusive, for membranes naturally enriched with a given protein such as retinal rod disks (Papermaster \& Dreyer, I974; Zimmerman, Daemen \& Bonting, 1976), sarcoplasmic reticulum (MacLennan \& Holland, 1975) or Halobacterium halobium (Henderson, 1977). This is, in general, rather exceptional since usually the protein of interest is a minor component of the membrane. However, the availability of specific functional assays of or known end points is immensely valuable in reconstitution studies; for example, substrates and inhibitors for enzyme systems, activators and 
inhibitors for receptors, or even mutants for specific proteins in bacterial systems. In this direction it has been recently possible to extract proteinlipid complexes from either mitochondria or submitrochondrial particles. These extracts are used to form protein-containing vesicles which display ATPase activity (Gomez-Puyou \& Darszon, 1980, unpublished results). Using the same methodology the $\mathrm{Mn}^{2+}$-dependent adenylate cyclase activity from sea-urchin sperm membranes has been reconstituted in vesicles (Darszon et al., unpublished observations).

With regards to the transfer mechanism of protein-lipid complexes into apolar solvents, it is evident that supplementary phospholipids and overall charge neutralization are required in order to favour the partition of functional proteins into solvents. (Montal, 1974; Darszon et al. 1978; Schönfeld et al. 1980). This process probably occurs through a phase inversion where the hydrophilic sections of the lipid and protein which interacted before with the bulk aqueous phase are enclosed in a polar core that traps water and is surrounded by phosphoilipid and/or surfactant acyl chains, which in turn are in contact with the apolar environment (Montal, 1974, 1976; Darszon et al. 1978; Schönfeld et al. 1980). This schematic view allows one to estimate the dimensions of the complex from its composition. The average molecular weight of a particle containing one protein plus water, lipid and/or detergent associated with it would be $\cong 6 \times 10^{6}$. The water content accounting for close to half the total weight, could be localized in a central droplet having a diameter of $0.022 \mu \mathrm{m}$. Most of the lipid present would fit in a single layer surrounding the surface of this particle, increasing its size to about $0.027 \mu \mathrm{m}$. The observed sizes of the majority of reaction centerlipid complexes in hexane were between $0.015 \mu \mathrm{m}$ and $0.085 \mu \mathrm{m}$ in diameter. This large distribution in particle size was accounted for by variations in water, lipid and protein content; the larger particles may contain more than one protein (Schönfeld et al. 1980).

The protein-lipid complexes in apolar solvents are the building blocks of several kinds of model membranes (see Fig. 2). In addition, they are interesting preparations in their own right. Studies of functional membrane proteins in low dielectric constant media which stimulate the membrane milieu are scarce. An attractive possibility to be explored is the development of new isolation and purification techniques for membrane proteins from protein-lipid complexes derived from complex membrane systems. The protein-lipid complexes in organic solvents could also be relevant and potentially informative model systems to 
explore alternative strategies aimed at achieving ordered structures and ultimately crystals.

The photochemical and functional analysis of rhodopsin and reaction centers that have been extracted as protein-lipid complexes into organic solvents and then reconstituted in model membranes, validates the notion that the full cycle of transferring a membrane protein from the native membrane into apolar media and back into a model membrane in aqueous solutions can be accomplished maintaining the biological activity of the protein.

(ii) Monolayer self-assembly from (protein-)lipid vesicle suspensions (strategies 2A-D, Fig. 2)

(1) Membrane formation (Schindler, 1980a). The technique is based on the spontaneous formation of a monolayer at the air-water interface of vesicle suspensions. The suspension $\left(0.5^{-1} \mathrm{mg} \mathrm{lipid} / \mathrm{ml}\right)$ is introduced into the two compartments of a membrane chamber to just below the aperture in the Teflon septum which separates the two compartments (Fig. 2). After a short period ( $\sim$ I $\mathrm{min}$ ) the water levels are successively raised above the aperture within which the two monolayers combine to form a stable planar bilayer (Montal \& Mueller, 1972). This success strictly depends on the use of sufficiently sized (radius $>50 \mathrm{~mm}$ ) unilamellar vesicles.

(2) Underlying processes (Schindler, 1979b). The above criterion for successful bilayer formation has been related to processes occurring during monolayer self-assembly at the surface of vesicle suspensions. The steady-state pressure in the monolayer was found to increase with increasing vesicle size and to reach a limiting value (termed 'equivalence

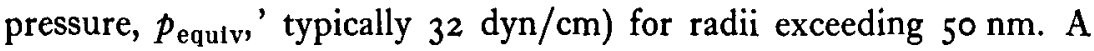
detailed analysis revealed an exchange equilibrium between monolayer and vesicles characterized by an equilibrium surface pressure, $p_{e}$. The vesicle size dependence of the success of bilayer formation resides in the size dependence of $p_{e}$ which was quantitatively attributed to the effect of vesicle size on the lipid exchange rate between vesicles and monolayer. For vesicle radii larger than $50 \mathrm{~nm}$ this limited size effect becomes negligible, the monolayer is in equilibrium with basically a planar bilayer, i.e. the state of lipids in the two environments is energetically equivalent. This explains why two such monolayers at $p_{\text {equiv }}$ can be combined into a stable planar bilayer. The equilibrium established 
between monolayer and vesicles as such (as a phase) was established from the independence of $p_{e}$ on vesicle concentration.

(3) Applicability for reconstitution work. The technique appears to be applicable to vesicles of any composition ranging from lipid vesicles to native biomembrane vesicles (see also Verger \& Pattus, 1976; Pattus, Desmuelle \& Verger, $1978 a$, and Pattus et al. 1978b) provided the vesicle radius is larger than $50 \mathrm{~nm}$. It thus allows to combine results obtained at different degrees of preservation of the original membrane conditions and studied at the three major configurations: vesicle, monolayer, planar bilayer. The primary parameters of reassembled planar membranes can be controlled: (a) membrane composition is determined from the quantitation of the composition of monolayers and adjusted by changing the composition of the vesicles in the aqueous phase, $(b)$ membrane energy is assayed by the equivalence pressure and modified by changing vesicle composition, $(c)$ initial distributions are imposed, $(\alpha)$ by forming two different monolayers (transverse distribution) and $(\beta)$ by using mixtures of vesicles with different composition (lateral distributions); for example, lipid vesicles mixed with lipidprotein vesicles containing either one or zero protein per vesicle (random distribution) or containing more than one protein per vesicle (nonrandom distribution).

(iii) Fusion of preformed (protein-)lipid vesicles with preformed planar bilayers

Another promising strategy to incorporate membrane proteins into planar bilayers is that of membrane fusion (strategies $3 \mathrm{~A}$ and B, Fig. 2). The initial reports of Skulachev and collaborators (Drachev et al. 1974), related to the mitochondrial $\mathrm{H}^{+}$-ATPase, cytochrome c-oxidase, bacterial reaction centres and bacteriorhodopsin showed that a genuine bilayer was not required since the measurements were reproduced on thick planar membranes. Therefore, such results should be distinguished as merely due to the adsorption of vesicles on to the planar film and not as incorporation of the protein into the bilayer (see Montal, 1976).

The approach of fusion was revived in order to study the permeability properties of the sarcoplasmic reticulum (SR). The experimental protocol consists of making SR vesicles interact with planar bilayers in the presence of a negatively-charged lipid, calcium, and osmotic conditions leading to the swelling of the vesicles (Miller \& Racker, 1976; Miller 
et al. 1976). Consequently, the bilayer conductance increased in discrete 'fusion events' and when the desired conductance level was attained, an excess of EDTA was added to stop fusion. Under favourable fusion conditions, the conductance increase is composed of a sequence of spike-like events of widely varying size. It was suggested that each of the above spikes represents the fusion of a single SR vesicle with a bilayer (Miller \& Racker, 1976; Miller, 1978) resulting in the insertion of a package of potassium channels. At high current resolution, the conductance rapidly fluctuates between discrete levels corresponding to the opening and closing of single channels: the single-channel conductance is $120 \mathrm{pS}$ in $0 . \mathrm{I} \mathrm{M}-\mathrm{K}+$ (Labarca, Coronado \& Miller, I980). Furthermore the steady-state conductance is voltage-dependent: at voltages more than $-60 \mathrm{mV}$ the conductance is low and as the voltage is shifted in the positive direction the conductance increases and eventually saturates at around $+15^{\circ} \mathrm{mV}$ (Labarca et al. 1980).

The channel characteristics are consistent with a model that considers that the channels have a single open and a single closed state, that they are independent and that the channel is a preformed permanent entity where the single-channel conductance is ohmic (Labarca et al. 1980).

An interesting feature of this channel is that it is symmetric but it is blocked by $\mathrm{Cs}^{+}$asymmetrically (only when added to the same compartment as the modifying material) (Coronado \& Miller, 1979). The Cs+ effect is manifested by a decrease of the open channel conductance and prolongation of the channel open time indicating that $\mathrm{Cs}^{+}$binds to the open channel shifting it to a blocked state. The functional role of this channel in the activity of the sarcoplasmic reticulum is suggested to be the provision of an electrical shunt that prevents the development of a large voltage across the membrane consequent to the flow of $\mathrm{Ca}^{2+}$ during release and re-uptake (Labarca et al. 1980).

A voltage-dependent anion channel (VDAC) from mitochondria has also been incorporated into decane-containing black lipid membranes by fusion (Cohen, Zimmerberg \& Finkelstein, 1980): osmotically lysed rat liver mitochondira were sonicated with a 25 -fold excess of phospholipids, lyophylized and resuspended in hexane. Multilamellar vesicles were prepared from the hexane extract by hydrating the residue left after the solvent evaporation. Such vesicles added to one of the aqueous compartments separated by a black film in the presence of an osmotic gradient across the planar membrane, with the side containing the vesicles hyperosmotic to the opposite side, led to the incorporation of 
VDAC into the planar membrane (Cohen et al. 1980). Divalent cations catalyse this process and therefore, the two requirements for fusion are an osmotic gradient across the membrane and the presence of divalent cations (Miller \& Racker, 1976; Zimmerberg, Cohen \& Finkelstein, I980). It is proposed that fusion occurs by the osmotic swelling of vesicles in contact with the planar membrane, with subsequent rupture of vesicular and planar membranes in the region of contact (Miller \& Racker, 1976; Zimmerberg et al. 1980; Cohen et al. 1980).

Another voltage-dependent anion channel was incorporated into solvent containing black lipid membranes. This material is derived from the electric organ of Torpedo californica (White \& Miller, 1979). When membrane vesicles rich in acetylcholine receptor ( $A C h R$ ) were added to one of the aqueous compartments separated by a black lipid membrane the membrane conductance increased. The conductance consisted of two components: an ohmic background and a voltage-dependent, ideally anion-selective conductance. The membrane conductance is due to the formation of channels with a single-channel conductance of $14 \mathrm{pS}$ in $0 . \mathrm{I}^{\mathrm{M}} \mathrm{Cl}^{-}$. Furthermore, the conductance-voltage curve is highly asymmetric: the anion conductance is low at voltages greater than $+10 \mathrm{mV}$ increases sharply as the voltage becomes negative and then saturates as the voltage becomes highly negative (the side of the bilayer to which the vesicles were not added, was defined as ground) (White \& Miller, 1979). The anion channel was inhibited by two well documented inhibitors of anion transport systems, SITS and DIDS (isothiocyanodysulphonic acid stilbene derivatives). In contrast, no cholinergic agents tested had any effect on the bilayer conductance: carbamylcholine, $d$-tubocurarine, procaine, or $\alpha$-bungarotoxin (White \& Miller, 1979). The origin and the function of the anion channel reported is uncertain (White \& Miller, 1979). It should be noted, however, that this anion channel derived from Torpedo has different characteristics to the voltagedependent anion channel of mitochondria (see II, C, p. 43), namely (I) the Torpedo anion channel is ideally anion selective while the mitochondrial channel shows only a 2 - to 7 -fold anion selectivity; (2) the single-channel conductance of the Torpedo anion channel is $14 \mathrm{pS}$ while that of VDAC is $45^{\circ} \mathrm{pS}$ (in $0 . \mathrm{I} \mathrm{M}-\mathrm{KCl}$ ); (3) the voltage-conductance curve of the Torpedo anion channel is asymmetric while that of VDAC is symmetric. Therefore, the two voltage-dependent anion channels appear to be different.

These three examples illustrate the difficulty in evaluating results 
obtained in reconstituted membranes in the absence of a sound physiological end point.

(iv) Incorporation of detergent-solubilized membrane extracts into preformed planar bilayers (strategy 3 , Fig. 2)

Historically speaking, this was the first strategy implemented and has been the one more extensively applied (for review, see Montal, 1976; Shamoo \& Murphy, 1979). However, the majority of studies reported non-specific disruptive effects which are initially manifested as discrete discontinuities that may even appear as discrete conductance fluctuations, but that eventually extend and disrupt the entire membrane. These adsorption-disruption events, when present in the absence of any other functional assay, have been a misleading contribution to the field. On the other hand, if this strategy is used in conjunction with others illustrated in Fig. 2, where no detergent is required for the reassembly process, it is possible, in principle, to exploit the inherent features of this sequential approach. We refer to this situation in the case of porin (Section, IIC) and of the mitochondrial anion channel (Section II, C).

\section{(II) Channels}

We now turn to describe in more detail functional reconstitution studies involving rhodopsin, acetylcholine receptor and porin. The studies on membrane proteins reconstituted in lipid vesicles are presented in detail because they may be used as the starting material to generate monolayers at the air-water interface and subsequently lipid bilayers by apposition of two monolayers. Moreover, this provides a unique opportunity to complement studies in lipid vesicles with studies in planar lipid bilayers of the same protein.

\section{(A) Rhodopsin}

(i) Background

The primary event in visual excitation is still considered to be the photoisomerization of retinaldehyde, the chromophore of the visual pigment rhodopsin, from the II-cis to the all-trans configuration (Rosenfeld et al. 1977). The photoisomerization initiates a sequence of conformation transitions in the protein moiety. It is now accepted that these light-induced changes in rhodopsin lead to cellular excitation by 
modulating the ionic conductance of the photoreceptor cell membrane (Hagins, 1972). However, the coupling mechanism of the rhodopsin photochemical changes to the cell electrical response is unknown (Hubbell \& Bownds, 1979; Montal, 1979).

Vertebrate rhodopsin has been solubilized and purified in several detergents (for reviews see Montal, 1979; Hubbell \& Bownds, 1979). Its molecular weight is around 37000 daltons and is composed of a protein moiety (opsin) with two oligosaccharides covalently bound near the amino terminus, and the chromophore, bound to opsin in the dark through a protonated almidine linkage. The $c$-terminus is located at the cytoplasmic surface of the disks and the amino terminus is believed to be exposed to the intradiscal aqueous medium. An important fraction of the protein is embedded within the membrane interior and its shape is asymmetric.

In vertebrate photoreceptors, phototransduction is thought to involve a diffusible internal transmitter which couples photon absorption by rhodopsin in the intracellular disk membranes to the $\mathrm{Na}^{+}$conductance decrease in the plasma membrane of the outer segment (Hagins, 1972; Baylor, Hodgkin \& Lamb, 1974; Baylor, Lamb \& Yau, 1979; Penn \& Hagins, 1972). The nature of the transmitter and its linkage to rhodopsin action is a subject of growing interest. Two leading hypotheses are currently being investigated.

(1) Hagins \& Yoshikami (1977), Yoshikami \& Hagins (1978) proposed that $\mathrm{Ca}^{2+}$ ions act as the internal transmitter: cytoplasmic $\mathrm{Ca}^{2+}$ activity increases during illumination because of a light-dependent increase in disk membrane permeability to $\mathrm{Ca}^{2+}$. $\mathrm{Ca}^{2+}$ regulates the ' $\mathrm{Na}^{+}$-dark current' by binding reversibly to the plasma membrane sites through which the $\mathrm{Na}+$ flows into the rod outer segment. They proposed no specific role for rhodopsin but an attractive possibility is that rhodopsin itself forms a transmembrane $\mathrm{Ca}^{2+}$ channel (Montal, Darszon \& Trissl, 1977).

The excitatory role of $\mathrm{Ca}^{2+}$ is supported by several lines of evidence which, briefly, indicate that the sensitivity and kinetics of the receptor light response are dependent on the intracellular $\mathrm{Ca}^{2+}$ concentration. Recent measurements with intact retinas directly deposited, photoreceptor side, over $\mathrm{Ca}^{2+}$-selective electrodes have shown $\mathrm{Ca}^{2+}$ releases up to ${ }_{10} \mathrm{Ca}^{2+} /$ bleached rhodopsin which rise to peak within $2-4 \mathrm{~s}$ (Gold \& Korenbrot, I980). Similar observations were made with $\mathrm{Ca}^{2+}$-selective microelectrodes, indicating that the $\mathrm{Ca}^{2}+$ fluxes are sufficiently large and 
occur with the time course to be expected from the $\mathrm{Ca}^{2+}$-hypothesis (Yoshikami, George \& Hagins, 1980).

(2) Cyclic GMP hypothesis. The plasma membrane $\mathrm{Na}^{+}$-conductance depends on the intracellular levels of cyclic GMP. Rhodopsin bleaching regulates cyclic GMP levels through the activation of a GTPase and a phosphodiesterase (Hubbell \& Bownds, r979; Woodruff \& Bownds, 1979; Miller \& Nicol, 1979; Bitensky et al. 1978). More specifically, it has been suggested that the $\mathrm{Na}^{+}$conductance is through a $\mathrm{Na}^{+}$channel which is modulated by cyclic GMP through a reversible phosphorylation (open channel)-dephosphorylation (closed channel) of one of its protein components (Polans, Hermolin \& Bownds, 1979).

The transmitter role of cyclic GMP is supported by several lines of evidence. Briefly; in freshly detached rod outer segments, a single absorbed photon leads to the hydrolysis of $\sim 10^{4}$ cyclic GMP molecules within 0.1 s (Woodruff \& Bownds, 1979) and in disk membrane suspensions to $4 \times 10^{5} / \mathrm{s}$ (Yee \& Liebman, 1978). Electrophysiological studies have shown that cyclic GMP depolarizes rods within milliseconds after being injected intracellularly and that it increases the latency of the light-induced hyperpolarization (Miller \& Nicol, 1979). Considering a turnover number of $800 \mathrm{~s}^{-1}$ for the phosphodiesterase (Miki et al. 1975) it was calculated that one photoexcited rhodopsin activates $\sim 500$ phosphodiesterase molecules (Liebman \& Pugh, 1979). The presence of the GTPase is necessary for the photoactivation of the phosphodiesterase (Liebman \& Pugh, 1979; Shinozawa et al. 1980; Fung \& Stryer, 1980) suggesting that the interplay between the membrane protein-rhodopsin and the peripheral proteins-GTPase and phosphodiesterase may be an important step in the phototransduction process.

In addition to the enzymic regulation of the C-GMP levels, there is a tight interrelation between the cytoplasmic levels of $\mathrm{Ca}^{2+}$ and cyclic GMP, such that increases in cyclic GMP levels or reduction in $\mathrm{Ca}^{2+}$ concentration produce similar alterations in the electrical activity of rods (Lipton, Rasmussen \& Dowling, 1977).

It has been suggested $\mathrm{Ca}^{2+}$ ions may be a means of coupling rhodopsin with the enzymes involved in the synthesis and degradation of cyclic GMP (Montal, 1979).

In addition, rhodopsin bleaching also activates the phosphorylation of opsin (cf. Hubbell \& Bownds, 1979). These findings bring photoreceptors together with other membrane receptors for neurotransmitters and hormones where the triad of $\mathrm{Ca}^{2+}$, cyclic nucleotides and 
receptor phosphorylation is characteristic and could be associated to the stimulus-activated transduction process (cf. Greengard, 1978).

The hypothesis that considers rhodopsin as a light-activated permeability pathway through which transmitter is released, can be directly tested in a membrane model system (Hubbell \& Bownds, 1979; Montal, 1979). The general strategy for the reassembly of a biologically active planar bilayer has been discussed in previous sections.

(ii) Vesicles

Photochemically active rhodopsin was incorporated into phospholipid vesicles (Hong \& Hubbell, 1972; Applebury et al. 1974; O'Brien et al. I $977 a$; and Darszon, Montal \& Zarco, 1977 $b$ ). The recombinant vesicles respond to light by releasing trapped ${ }^{22} \mathrm{Na}^{+},{ }^{134} \mathrm{Cs}^{+},{ }^{45} \mathrm{Ca}^{2+}$, glycerol and glucose (Darszon et $a l$. $1977 b$ ). Sucrose and inulin remained trapped, indicating that the light-induced permeability pathway had a cut-off diameter of around ro $\AA$. The wavelength dependence of the lightinduced release of ${ }^{22} \mathrm{Na}^{+}$and ${ }^{45} \mathrm{Ca}^{2+}$ correlated, within experimental error, with the absorption spectrum of rhodopsin (Darszon et al. 1977b). Flash illumination, which bleached approximately $80 \%$ of rhodopsin, released all the trapped ${ }^{45} \mathrm{Ca}^{+}$; (Hubbell et al. 1977). These results were confirmed with NMR techniques where a light-induced permeability increase to $\mathrm{Mn}^{2+}, \mathrm{Eu}^{2+}$ and $\mathrm{Co}^{2+}$ was observed (O'Brien et al. $1977 b$ ). Therefore light induces the formation of a poorly selective permeability pathway in rhodopsin-phospholipid vesicles. Although, this supports the hypothesis that considers rhodopsin as a light-induced channel, serious questions concerning the kinetics and efficiency of the response remain to be answered.

\section{(iii) Monolayers and planar bilayers}

There are several reports describing photoresponses of black lipid films modified with rhodopsin. Unfortunately, no definite conclusions can be stated until these systems are adequately characterized (Takagi, Azuma \& Kishimoto, 1965; Takagi, I980; Fesenko, Ratner \& Lyvbarsky, 1976, Fesenko \& Lyvbarsky, 1977; Antanavage et al. 1977).

As described before it is now possible to transfer into organic solvents rhodopsin-lipid complexes (RLC) derived from both detergent-solubilized and affinity-purified rhodopsin (Darszon et al. 1978; Darszon, Blair \& Montal, 1979) as well as directly from membrane-bound rhodopsin (Darszon et al. 1978; Darszon, Montal \& Philipp, 1977). 
In order to assemble rhodopsin-containing bilayers, the RLC in the solvent is spread at an air-water interface. The competence of this interfacial layer was evaluated to assure that rhodopsin was photochemically active at each step of the reconstitution process (Trissl, Darszon \& Montal, 1977). After spreading the RLC in ether at an airwater interface the layer was apposed onto one side of a thin $(\sim 6 \mu \mathrm{m})$ Teflon film which separates two aqueous compartments and acts as an electrical insulator coupling the compartments capacitatively (Trissl et al. 1977). Flashes evoked fast photoelectrical signals (1 ms) that originate from capacitative charge displacements of orientated rhodopsin as it goes through the conformational transitions upon bleaching. The peak amplitude of the photocurrent was proportional to the number of unbleached rhodopsin, in a similar manner to the early receptor potential (ERP) recorded in albino rat retinas (Cone \& Pak, 1971). The action spectrum of the photoresponse (model ERP) agrees with the absorption spectrum of rhodopsin. Therefore, these displacement photosignals establish the presence of excitable and oriented rhodopsin molecules at the interface and also that the ERP of photoreceptor cells and the model ERP originate from capacitative charge displacements in rhodopsin (Trissl et al. 1977; Trissl, 1979; Chapron, 1979). Similar results were obtained by directly spreading retinal rod disk membranes at the air-water interface (Trissl et al. 1977; see Fig. 2, strategy 2D).

Planar bilayers formed from RLC in hexane or ether had an absolute membrane conductance (measured under voltage-clamp conditions) in the range of $0 \cdot 2-2 \cdot 0 \mathrm{nS}$ varying from one membrane to another. Regardless of the dark conductance level, the light response was usually an increase in membrane conductance (Montal, 1975; Montal et al. 1975, 1977). Two salient points are noteworthy: (1) a distinct latency period between illumination and onset of the conductance change: it varied from a second to several hundred seconds and appeared shorter with increasing concentrations of rhodopsin in the extract (Montal et al. 1977); (2) the light-induced conductance change is irreversible: when a membrane is illuminated and responds, the conductance increase continues even if the light is turned off during the transition from low to high conductance.

A characteristic feature of the steady-state conductance of rhodopsincontaining bilayers is the occurrence of defined fluctuations. Three main transitions appear in symmetric solutions of $0.2 \mathrm{M}-\mathrm{KCl}$ (or $\mathrm{NaCl}$ ), with amplitudes of about 200,400 and $600 \mathrm{pS}$; smaller transitions of 
20 and $40 \mathrm{pS}$ also occur (Montal et al. 1977). Illumination increases the absolute conductance and the frequency of transitions but does not change the fluctuation pattern. These results as well as those obtained from the rhodopsin vesicles experiments are consistent with the lightinduced formation of a channel in rhodopsin-containing bilayers (Montal, 1975; Montal et al. 1975, 1977).

The results hitherto described were obtained with preparations of rhodopsin-lipid complexes in hexane or ether derived from both detergent-solubilized as well as from membrane-bound rhodopsin (Darszon et al. 1978). Although rhodopsin is the major protein component of rod disks ( $>80 \%$ ) (Hubbell \& Bownds, 1979), it is conceivable that other disk proteins could contribute to the bilayer results. In addition, the lipids from the disks are also extracted into the solvent and since they are extremely unsaturated $(50 \%$ of the fatty acids are docosahexanoic acid) there is concern that their oxidation products would play a role in the formation of channels (Darszon et al. 1979a).

To assess this possibility it was shown that affinity-purified and delipidated rhodopsin recombined with a single non-oxidizable phospholipid, or a lipid mixture of known composition, was efficiently transferred as a lipid-protein complex into organic solvents. The absorption spectra, stability and regeneration capacity of rhodopsin from these preparations indicated that the overall photochemical behaviour of the protein was preserved throughout the procedure. Furthermore, planar bilayers formed from this material (Darszon et al. r $979 a$; Ramakrishnan et al. 1980) displayed channel activity with similar characteristics as those reported before (Montal et al. 1977).

The similarity of the voltage-dependent channel activity observed in rhodopsin-containing bilayers and in VDAC (see II, C) (voltagedependent anion channel from mitochondria Schein et al. 1976; Colombini, 1979, 1980), modified lipid bilayers, has raised the question that contamination of rod disk membranes with mitochondria could account for the bilayer results. There are at least five salient arguments against this contention: ( $I$ ) the light-dependence of the rhodopsinbilayer conductance (Montal et al. 1977); (2) the fact that the same channel activity can be recorded in bilayers generated from extracts derived from rod disk membranes (Montal et al. 1975, 1977) or from detergent-solubilized and affinity-purified rhodopsin (Darszon et al. I 979a); (3) the apparent pore size of Neurospora crassa VDAC- and of rhodopsin-proteoliposome is 40 and 1o $\AA$, respectively: VDAC allows 
the free-diffusion of inulin (Stokes-Einstein radius of ${ }_{14} \AA$, Scherrer and Gerhart, 1971) (see Colombini, 1980); in contrast, the permeability to sucrose ( $\sim$ IO $\AA$ in diameter) and inulin was not modified by light excitation of rhodopsin-lipid vesicles (Darszon et al. 1977b; Hubbell et al. 1977); (4) the channel activity associated to VDAC can be recorded in decane-containing black lipid films (Cohen et al. 1980) while that of rhodopsin cannot (Montal et al. 1977; cf. Montal, 1979 (5) the VDAC-induced conductance fluctuations are of uniform size $(4.5 \mathrm{nS}$ in $\mathrm{I} \cdot 0 \mathrm{M}-\mathrm{KCl}$, Colombini, $1979, \mathrm{I} 980$ ) whereas those associated with rhodopsin always occur in multiples, e.g. 200, 400 and $600 \mathrm{pS}$ in $0.2 \mathrm{M}-\mathrm{KCl}$ (Montal et al 1977; cf. Montal, 1979). Thus, the channel activity recorded in planar bilayers formed from purified rhodopsin and a non-oxidizable phospholipid cannot be attributed to lipid oxidation and the possibility of assigning the channel activity to a non-rhodopsin protein component has been rendered unlikely. Therefore, the interpretation that the channels are associated with the presence of rhodopsin in the bilayers is still favoured (Darszon et al. 1979a).

Based in this conclusion the analysis of the ion-channel in rhodopsincontaining planar lipid bilayers proceeded. It was previously shown that, under voltage-clamp conditions, the membrane current fluctuated in discrete events, consistent with the model of a channel opening and closing. The individual conductance fluctuations were ohmic but the overall conductance of membranes with many channels was voltage sensitive and became lower as the voltage was increased (Montal et al. 1977). Voltage-jump relaxation experiments showed that the channels open at zero voltage and close at higher voltages (Montal et al. 1977; Ramakrishran et al. 1980). However, the relaxation of the conductance was not a single exponential indicating that either the opening or the closing of the channel, or both, are not simple Poisson processes.

Analysis of single-channel membranes provided clues to the mechanism of channel formation and operation. It was found that the distribution of channel dwell times in the open state is well described by a single exponential, indicating that the closing of the channel is Poissonian. This was taken as evidence that the open state of the channel is a single state. In contrast, there was significant deviation from a single exponential of the closed dwell times. This indicated the presence of more than one closed state (Ramakrishnan et al. I980). The results are consistent with the hypothesis that channel formation proceeds by aggregation of rhodopsin monomers and that channel 
closing can occur by a simple dissociation of the aggregate (Montal et al. 1977; Ramakrishnan et al. 1980).

The initial hint for the involvement of subunit aggregation in the process of channel formation was derived from the observed long latency period (seconds) between application of the light stimulus and the bilayer photoresponse (Montal et al. 1977). The latency period could be accounted for by the delay in the assembly of a channel from precursors, namely rhodopsin monomers: consider that the effect of light is to populate the membrane with bleached rhodopsin units which subsequently aggregate with other bleached or unbleached units by laterally diffusing in the plane of the bilayer (Montal et al. 1977). For an aggregation mechanism to be valid it would be expected to find a high order concentration dependence of both the steady-state conductance amplitude as well as for the time constants of the conductance change. This expectation is not met satisfactorily by experiments since there is no way to ascertain the rhodopsin concentration in the bilayer and the concentration in the extract is not representative of that present in the surface layers. Furthermore, it is likely that there are phase separations in the monolayers into protein-enriched and proteinfree areas, that when apposed through the aperture to form a bilayer give results representative of the area sampled.

Thus, in an effort to overcome this problem it became desirable to incorporate rhodopsin into spherical bilayers of large size in order to apply electrophysiological techniques and take advantage of a large inner volume as well as to correlate the properties with rhodopsin content. It was shown that rhodopsin-lipid complexes in hexane or ether could be reassembled into single bilayer vesicles of about I $\mu \mathrm{m}$ in diameter (ref. Darszon et al. 1979c). This large size is still small relative to the size of microelectrodes one can routinely use for electrophysiological studies. Therefore, attempts were pursued to produce larger vesicles, as large as $30 \mu \mathrm{m}$ (average diameter) (ref. Darszon et al. 1980). The optical spectral properties of rhodopsin in the large vesicles were similar to those in native rods; furthermore, rhodopsin in the vesicles was chemically regenerable with 9 -cis retinal (Darszon et al. 1980). Freeze-fracture replicas of the vesicles revealed that both internal and external leaflets contained numerous intramembranous particles of about $80 \AA$ in diameter. The large vesicles were penetrated with microelectrodes and visualized by the injection of a fluorescent dye. Preliminary electrical recordings were obtained by sealing the vesicles 
to a hole in the septum separating two aqueous compartments (Antanavage et al. 1978; Darszon et al. 1980). This now opens the way to study the properties of lipid bilayer vesicles containing purified rhodopsin by chemical, optical and electrical techniques.

A working hypothesis that emerges from considering the biochemistry and physiology of the photoreceptor and the results obtained with rhodopsin in model membranes, attributes the modulation of the photoreceptor plasma membrane ion permeability by photolysed rhodopsin to a light-activated and voltage-sensitive transmembrane channel formed by rhodopsin aggregation (Montal, 1975; Montal et al. 1977). A possible sequence of events leading to excitation could be: in the dark the channel is closed and $\mathrm{Ca}^{2+}$ is sequestered inside the disks by an ATPase; or a GTPase (Robinson \& Hagins, 1979) or a $\mathrm{Ca}^{2+} / 2 \mathrm{Na}^{+}$antiport (Schnaetkamp, 1980). Exposure to light induces channel formation allowing $\mathrm{Ca}^{2+}$ to diffuse down its concentration gradient. The diffusion of $\mathrm{Ca}^{2+}$ through the disk membrane would change the membrane potential to a value at which the channel closes. These changes of $\mathrm{Ca}^{2+}$ concentration in the cytoplasm together with the light activated GTPase and the phosphodiesterase can regulate the cytoplasmic cyclic GMP levels, which in turn control the $\mathrm{Na}^{+}$plasma membrane conductance the underlying mechanism also remains to be solved (Hubbell \& Bownds, 1979; Montal, 1979). An attractive aspect of this working hypothesis is its susceptibility to be tested. Reconstitution techniques are rapidly making progress and their use in unravelling the sequence, interrelation and importance of the events that occur in phototransduction appears promising.

\section{(B) Acetylcholine receptor}

(i) Background

Transmission of signals from motoneurons to striated muscle fibres at the neuromuscular junction is mediated through nicotinic acetylcholine receptors (AChR) located in the post-synaptic membrane. The acetylcholine receptor is responsible to transduce the binding of the transmitter acetylcholine ( $\mathrm{ACh}$ ) into an increase in the permeability of the post-synaptic membrane to both sodium and potassium ions (for detailed reviews see Rang, 1975; Gage, 1976; Karlin, 1980). The AChR is by far the best characterized neurotransmitter receptor, both physiologically as well as biochemically, and therefore, it provides an archetype 
for understanding the structure and function of signal-transducing membrane proteins.

The AChR has been isolated in the form of AChR-rich membrane fragments, many of which exist as closed vesicles (Karlin, 1980; Heidemann \& Changeux, 1978; Briley \& Changeux, 1977). Using vesicles both the binding of cholinergic ligands and agonist-induced cation permeability can be studied (e.g. Neubig \& Cohen, 1980). The $\mathrm{AChR}$ has also been solubilized with detergents and purified by affinity chromatography to homogeneity (Raftery et al. 1975; Karlin et al. 1976; Lindstrom \& Patrick, 1974). Amino acid sequence analysis of the purified subunits has started (Raftery et al. 1980; Devillers-Thiery et al. 1979) and high resolution structural studies are currently under way (Zingsheim et al. I980; Klymkowsky, Heuser \& Stroud, I980).

The AChR from Torpedo californica is an integral membrane glycoprotein of which the monomeric form is composed of four glycopeptide subunits termed $\alpha, \beta, \gamma$ and $\delta$ with apparent molecular weights approximating 40000, 50000, 60000 and 65000 daltons (Reynolds \& Karlin, 1978; Lindstrom et al. 1979; Raftery et al. 1980). Several lines of evidence indicate that the subunit stoichiometry is $2: 1: 1: I$ implying that the protein is a pentameric complex with a molecular weight of 255000 daltons (cf. Karlin, 1980; Lindstrom et al. 1979; Raftery et al. 1980). The $\alpha$ subunit is known to contain part or all the acetylcholinebinding sites (Weill, McNamee \& Karlin, I974). However, the exact functions of the other subunits are still unknown. It appears, that $\beta, \gamma$ and $\delta$ subunits are very protease sensitive while $\alpha$ is relatively resistant, and that the nicked subunits remain closely associated and functional (Lindstrom et al. $1980 \mathrm{~b}$ ). These results indicate that the AChR subunits are intimately associated by noncovalent interactions and not readily disassociated even by proteolysis. In agreement with this, it has been shown that a photoaffinity labelling reagent coupled by a long side arm to $\alpha$-bungarotoxin can link the toxin to any of the AChR subunits. This suggests that in the membrane all of the AChR subunits must be relatively close to one another (Nathanson \& Hall, 1980). Thus, the currently accepted view is that the AChR is a pentameric protein complex composed of four distinct and homologous subunits that appear to be responsible for the activity of the receptor (Lindstrom et al. 1980a; Raftery et al. 1980; Karlin, 1980).

Whereas electric organ AChR have been extensively studied biochemically, muscle AChR have been intensely investigated electro- 
physiologically (Gage, 1976; Fambrough, 1979). The physiological characteristics of the AChR-mediated conductance increase occurring in post-synaptic membranes have established that each functional AChR has two binding sites on the extracellular surface (Dionne, Steinbach \& Stevens, 1978). Two acetylcholine molecules bind rapidly to the resting closed channel to form a receptor-acetylcholine complex. It is generally presumed that agonist binding induces a conformation change in the AChR molecule which results in opening of a cationspecific channel. The complex undergoes a change in its conformation that opens a channel to the passage of both sodium and potassium ions. The channel appears to be at least as large as a square of 6.5 by $6.5 \AA$ (Watanabe \& Narahashi, 1979; Dwyer, Adams \& Hille, I980; Adams, Dwyer \& Hille, 1980). The channel remains open for about $1 \mathrm{~ms}$ (Neher \& Sakmann, 1976). The most compelling evidence for the existence of the channel in the post-synaptic membrane is derived from single-channel recordings of individual current steps generated by the opening and closing of individual channels (Neher \& Sakmann, 1976; Sakmann, Patlak \& Neher, I980). Single-channel recordings have provided a means to directly test many of the inferences about ionic channels which were derived from electrical noise analysis (Katz \& Miledi, 1972; Anderson and Stevens, 1973). These experiments have shown that the difference in conductance between open and closed states of the agonist-induced AChR channels is between $20-30 \mathrm{pS}$ and the energies are such that the channel dwells in the open state for a few milliseconds (Neher \& Sakmann, I976; Sakmann et al. I980). It is also known that prolonged exposure of AChR to agonist generates a desensitized conformation characterized by a high agonist affinity state with loss of responsiveness (channel closure). This desensitized state relaxes over seconds to the resting state (Katz \& Thesleff, 1957; Sakmann et al. 1980; Neubig \& Cohen, 1980). The lifetime of a channel is voltage-dependent such that the probability of closing the channel varies over an $e$-fold range for every $80 \mathrm{mV}$ change in membrane potential. The voltage dependence is attributed to differences in the dipole moment of open and closed states of the channel, respectively (Anderson \& Stevens, 1973; Magleby \& Stevens, 1972).

The goal of reconstitution is to reassemble the purified AChR into lipid bilayer membranes in an attempt to directly answer questions which are otherwise not readily accessible to experimental evaluation in the native system. For example, does purified receptor contain all the components necessary for function? Do the different types of subunits of 
purified AChR represent different functional entities? And if so, what is the interaction between them ? If the subunits could be selectively removed or separated without irreversible denaturation and reconstituted separately or in various combinations the answer to these questions could be foreseen. Furthermore, which subunit is responsible for the ion selectivity and voltage sensitivity of the channel, or are these properties associated to the channel entity? What is the role of surface charges and membrane asymmetry in AChR responses? What is the lipid dependence of channel function ? Moreover, one can gain experimental control over parameters which are not readily accessible or highly variable in the natural membrane. For example, one would like to control the ionic composition of the bathing medium on both sides of the membrane inlaid with the channel.

\section{(ii) Vesicles}

The search for procedures to reproducibly reconstitute the agoniststimulated cation conductance of AChR into planar lipid bilayers or lipid vesicles has been characterized by frustration and failure (reviewed by Montal, 1976; Heidmann \& Changeux, 1978). This may stem, among other factors, from the difficulty in preserving the native state of both the agonist-binding and channel components of the AChR. Epstein \& Racker (1978) showed that if membranes rich in AChR were solubilized in cholate-lipid mixtures and then the cholate was removed by dialysis, the vesicles formed exhibited a rapid, carbamylcholine-dependent uptake of ${ }^{22} \mathrm{Na}^{+}$, which was inhibited by $\alpha$-bungarotoxin and several other known inhibitors (Epstein \& Racker, 1978). Desensitization was exhibited by the reconstituted vesicles, readily demonstrated by preexposing the vesicles to carbamylcholine before adding radioactive sodium. These results indicate that when AChR is solubilized and purified in cholate or other detergents the cation channel is inactivated, but solubilization and purification in cholate soybean lipid mixtures preserved the activity of the ion channel (Lindstrom et al. 1980a; Huganir, Schell \& Racker, I979). Alkaline extraction ( $\mathrm{pH}=1 \mathrm{I} \cdot 0$ ) of highly purified membrane fragments from Torpedo californica (Wu \& Raftery, 1979) and Torpedo marmorata (Changeux et al. 1979) produces membrane fragments containing only the four polypeptide subunits characteristic of detergent-solubilized purified AChR (Neubig et al. 1979). These purified membranes were solubilized in cholate plus soybean phospholipid and then reconstituted into vesicles by removing 
the cholate via dialysis (Epstein \& Racker, 1978). The AChRs in the reconstituted vesicles display carbamylcholine-sensitive sodium flux (Wu \& Raftery, 1979; Changeux et al. 1979), showing that only the subunits present in purified AChR are necessary for AChR function.

The initial reconstitution experiments were performed with a crude mixture of soybean phospholipids (Epstein \& Racker, 1978). It was then shown that removal of neutral lipids from asolectin or replacement of asolectin with a mixture of pure phospholipids yielded vesicles that were less active in catalysing carbamylcholine-sensitive sodium flux. However supplementing the purified phospholipids with $\alpha$-tocopherol, coenzyme QIO or vitamin $\mathrm{KI}_{\mathrm{I}}$ during the reconstitution procedure led to vesicles which were considerably more active in the sodium influx assay than the vesicles reconstituted with crude phospholipid mixtures (Kilian et al. I980). This fact appears not to be due to the antioxidant properties of $\alpha$-tocopherol since other antioxidants were ineffective. It was suggested that the $\alpha$-tocopherol or the quinones were modifying the packing arrangements of the phospholipid bilayers (Kilian et al. 1980).

These initial studies were rapidly followed by the successful reconstitution of purified acetylcholine receptor into lipid vesicles. Huganir et al. (1979) solubilized AChR in cholate lipid mixtures and then purified it on a choline carboxymethyl affinity resin (Reynolds \& Karlin, 1978). The receptor complex was eluted with carbamylcholine and then reconstituted into vesicles by cholate dialysis. Reconstitution by cholate dilution or by freeze-thaw sonication (Kasahara \& Hinkle, 1976) were also used but they gave lower sodium influx values than the cholate dialysis procedure (Huganir et al. 1979). The carbamylcholinesensitive sodium influx of reconstituted vesicles was inhibited by $\alpha$ bungarotoxin and by the local anaesthetic procaine. They also displayed desensitization. Lindstrom et al. (1980a) purified AChR in cholate lipid-lipid mixtures by several methods involving both affinity chromatography (with Naja naja siamensis toxin or concanavalin A coupled to agarose) and non-affinity techniques (sucrose gradient centrifugation). The best method combined affinity chromatography on toxin-agarose and concanavalin A agarose. Receptors purified by all these methods were incorporated into lipid vesicles by cholate dialysis. The carbamylcholine-regulated cation channels of the reconstituted AChR were equally active, irrespective of the method used for purification, showing that exposure to antagonists like toxin and benzoquinonium during purification did not irreversibly alter AChR function. All reconstituted 
vesicle preparations contained the four subunits characteristic of affinity-purified AChR (Lindstrom et al. 1980a).

Torpedo AChR normally occurs as dimers formed by two monomers linked via a disulphide bond between their delta subunits (Chang \& Bock, I977; Hamilton, McLaughlin \& Karlin, 1979). Treatment of AChR dimers with reducing agents ( $\beta$-mercaptoethanol) converts dimers into monomers. Receptor monomers and dimers were separately reconstituted into soybean lipid vesicles by the cholate dialysis technique. Reconstituted monomers and dimers were functionally equivalent with respect to their carbamylcholine induced, dose-dependent uptake of ${ }^{22} \mathrm{Na}^{+}$, the total flux of ${ }^{22} \mathrm{Na}^{+}$per receptor during the permeability response, and the occurrence of desensitization (Anholt, Lindstrom \& Montal, 1980). Furthermore, it was shown that monomers were not non-covalently associated in their reconstituted vesicles, because reconstituted monomers, unlike reconstituted dimers, could not be efficiently covalently cross-linked by glutaraldehyde. These results, strongly suggest that both the acetylcholine-binding sites and the agonistregulated cation specific channel are contained within the $\alpha-2, \beta, \gamma$, $\delta$ subunit structure of the AChR monomer (Anholt et al. 1980). In the reconstituted vesicles more than $79 \%$ of the total $\alpha$-burgarotoxinbinding sites face outward (Huganir et al. 1979, Lindstrom et al. 1980a; Gonsalez-Ross, Paraschos \& Martinez-Carrion, I980). This asymmetric distribution of $\mathrm{AChR}$, similar to that of native membranes, has been exploited in the studies with planar lipid bilayers as will be mentioned later (Nelson et al. I980; Schindler \& Quast, I980).

All the studies detailed before have in common the use of cholate as the solubilizing detergent. It has been recently reported that AChR could be also solubilized and purified in octylglucoside, and then reconstituted into lipid vesicles by adding Torpedo lipids and removing the detergent by dialysis (Gonsalez-Ross et al. 1980). In contrast, octylglucoside added to cholate-lipid-solubilized AChR prevents reconstitution of carbamylcholine-induced ${ }^{22} \mathrm{Na}^{+}$uptake (Anholt, Lindstrom \& Montal, unpublished observations; Huganir \& Racker, personal communication). Gonsalez-Ross et al. (r980) used more than 8o-fold higher AChR to lipid ratios for reconstitution than the other groups. Thus, it appears that residual amounts of active AChR remaining after exposure to octylglucoside could account for the carbamylcholine-induced ${ }^{22} \mathrm{Na}+$ influx reported.

The studies with AChR reconstituted in lipid vesicles show that, in 
contrast with the situation several years ago (for a review see Montal, 1976, Briley \& Changeux, 1977) AChR reconstitution is now a reproducible, easy and efficient process. As long as the cation channel is protected by cholate-lipid mixtures several purification methods yield active $\mathrm{AChR}$ incorporated in lipid vesicles.

\section{(iii) Planar bilayers}

We now turn to a consideration of the recent successful reconstitution of AChR in planar lipid bilayers. These reports must be distinguished from previous claims of recovery of functional activity from black lipid films modified with incompletely characterized membranes or illdefined membrane extracts (for review see Montal, 1976; Heidmann \& Changeux, 1978; Karlin, 1980).

AChR has been incorporated into planar lipid bilayers essentially by the same procedure: monolayers at the air-water interface were spontaneously generated from a vesicle suspension, and thereafter a planar lipid bilayer was assembled by apposition of two such monolayers (see Fig. 2; Schindler \& Quast, 1980; Nelson et al. 1980). Two sets of preparations were used for the characterization of the AChR in planar lipid bilayers: ( $\mathrm{I}$ ) native membrane vesicles rich in AChR from Torpedo marmorata (Schindler \& Quast, I980); (2) reconstituted vesicles containing $A C h R$ which had been previously solubilized in lipid-cholate mixtures and purified by affinity chromatography using toxin-agarose and concancalin A-agarose affinity columns (Nelson et al. I980; Lindstrom et al. 1980a; Anholt et al. 1980).

In the experiments performed with native membrane vesicles (Schindler \& Quast, I980) bilayers were formed from mixed vesicle suspensions containing native and pure lipid (soybean lecithin :cholesterol, $6: 1$ ) vesicles in various ratios. The ratios of $A C h R$ to lipid in the monolayers were shown to directly correspond to those in the mixed vesicle suspension. This gave quantitative control of the AChR concentration in the monolayers and thus in the bilayers. The activity of AChR in the native vesicles was established by tracer flux experiments. Preservation of AChR function in the planar membrane was demonstrated by several criteria. (I) In the absence of agonist the membrane conductance was almost a low as that of lipid membranes. Addition of carbamylcholine caused a large steady state conductance which could be competitively inhibited by $d$-tubocurarine (Jenkinson, 1960). Competition was in quantitative agreement with electrophysiological results. 
Upon preincubation of native vesicles with $\alpha$-bungarotoxin no conductance increase was elicited by carbamylcholine. (2) Completely asymmetric membranes could be formed with respect to functional ligandbinding sites but the conductances were invariant to voltage reversal (no rectification). It is interesting to note that this asymmetry was found in spite of the incomplete sidedness of $A C h R$ in the native vesicles (see Hartig \& Raftery, 1979). (3) The time course of the conductance after a single dose of $\mathrm{I} \mu \mathrm{M}$ carbamylcholine showed a pronounced maximum. This maximum together with half saturation of conductance increase between 50 and $100 \mathrm{nM}$ carbamylcholine indicates that AChR can assume a desensitized state also in the planar membrane. It was further evidenced (Schindler, $1980 b$ ) that the peak and waveform of the response maximum is in quantitative agreement with kinetic data obtained on native vesicles assuming the current minimum model for the states of the AChR. (4) At low concentrations of native vesicles with respect to lipid vesicles the planar membranes exhibited carbamylcholine-induced single-channel fluctuations. Opening and closing of channels was evident from the observed discrete conductance events of $90 \pm 10 \mathrm{pS}$ at $\mathrm{I} \mathrm{M}-\mathrm{NaCl}$ and $20-25 \mathrm{pS}$ at $250 \mathrm{~mm}-\mathrm{NaCl}$. Channel opening times ranged between I and 2 msec. (5) The carbamylcholineinduced conductance was cation selective, about 7 times higher for potassium and sodium ions over chloride ions. These permeability ratios were measured in the presence of at least 100 channels and determined from Nernst potentials. (6) The quantitation of the AChR concentration in the monolayer which likely corresponds to that in the bilayer together with the number of observed channels at steady state versus those expected to be in the desensitized state allowed the estimation of the percent recovery of functional AChR in the membrane. The values ranged from 25 to about $\mathrm{I} \%$. The variability was indicated to reside in differences between the fish before death or deterioration of the electric organs with longer storage.

All this evidence led the authors to conclude that the observed conductance properties were due to the chemical activation of the AChR-associated channel. Thus, the transformation of native acetylcholine receptor vesicles to planar bilayers seems to conserve the expected properties of the receptor.

We now turn to describe the results obtained with purified AChR. Monolayers at the air-water interface were self-assembled from the reconstituted vesicles and planar bilayers were subsequently formed by 
apposition of two monolayers. These results are fundamentally analogous to those described for planar bilayers derived from native vesicles (Nelson et al. 1980; Schindler \& Quast, 1980). It was observed that addition of carbamylcholine to one of the aqueous compartments caused a large increase in membrane conductance, which spontaneously relaxed. This decay is consistent with receptor desensitization, a wellestablished physiological phenomenon (Katz \& Thesleff, 1957). The planar bilayers displayed high sensitivity in that concentrations of carbamylcholine as low as $25 \mathrm{nM}$ were sufficient to elicit a measurable change in conductance. The desensitization process could also be enhanced by addition of an excess of carbamylcholine at the peak of the conductance response. Thus, planar bilayers containing purified AChR displayed dose-dependent, spontaneously relaxing increases in conductance. Furthermore, the carbamylcholine-induced increase in membrane conductance could be inhibited by curare (Jenkinson, I960; Nelson et al. 1980). Asymmetric bilayers formed by apposing two monolayers composed of AChR-lipid and lipid without AChR showed asymmetric responses to carbamylcholine. When carbamylcholine was applied to the compartment limited by the lipid monolayer small or no changes in conductance resulted, but subsequent addition of the same concentration of agonist to the AChR-lipid side then elicited the characteristic response. In the reconstituted vesicles about $70 \%$ of the total $\alpha$ bungarotoxin-binding sites face outward (Lindstrom et al. 1980a). This preferred orientation appears to be preserved in the monolayer and in the planar bilayer, because the ratio of response amplitudes in the asymmetric bilayer was $\leq 6$-fold higher in the compartment containing the AChR-reconstituted vesicles (Nelson et al. 1980). Furthermore, this experimental strategy provides a built-in control as far as discarding unspecific pharmacological effects.

The carbamylcholine-induced increase in membrane conductance was always accompanied by an increase in the conductance noise. Fluctuation analysis indicated a single-channel conductance of $16 \pm 3 \mathrm{pS}$ (in $0 . \mathrm{I} \mathrm{M}-\mathrm{NaCl}$ ) with a mean channel open time estimated to be $35 \pm 5 \mathrm{~ms}$ (Nelson et al. 1980 ). Furthermore, in stable membranes that had low conductance, it was possible to resolve defined conductance fluctuations between discrete levels; these transitions were of approximately constant amplitude but varying duration. The single-channel conductance recorded with an applied voltage of $+10 \mathrm{mV}$ was $60 \mathrm{pS}$ $(0.5 \mathrm{M}-\mathrm{NaCl})$. The estimated mean channel open time was approxi- 
mately $50 \mathrm{~ms}$. Although these values were preliminary, they appear to be in fair agreement with the values estimated by fluctuation analysis (Nelson et al. 1980). When the reconstituted vesicles containing purified AChR are used to prepare membranes as described by Schindler \& Quast (1980), single channels with a conductance of $90 \pm 15 \mathrm{pS}$ and a mean open time of about $5 \mathrm{~ms}$ at an applied voltage of $100 \mathrm{mV}$ (in $\mathrm{I} \cdot \mathrm{M}-\mathrm{NaCl}$ without sucrose) were recorded (M. Montal and $\mathrm{H}$. Schindler, unpublished experiments).

One of the great advantages of this strategy of planar bilayer assembly is that it allows the correlation of results obtained in vesicles with those obtained in planar lipid bilayers at different levels of preservation of the native AChR environment. Furthermore, the functional integrity of native or purified AChR reconstituted in lipid vesicles can be assessed prior to planar bilayer formation; this provides the necessary confidence control that the starting material for planar bilayer assembly is indeed functionally active. Moreover, the fact that no further manipulations are required in order to assemble the planar bilayer except the plain fact of allowing the spontaneous self-assembly of a monolayer from the native or reconstituted vesicle suspension provides a strategy with a minimum number of perturbations. The bilayer vesicles are convenient for biochemical studies, radiochemical flux measurements (Hess, Cash \& Aoshima, 1979) and even for spectroscopic studies. The planar bilayer, in contrast, allows the study of the kinetics of the AChR response to agonists up to the microsecond time range and, therefore, enables investigation of the fine details of the response. Hence, it emerges that planar bilayers in conjunction with reconstituted vesicles constitute a powerful approach for investigating the mechanism of signal transduction of the AChR at the molecular level.

These two studies represent a promising starting point for the investigation of AChR in planar bilayers. Further adjustment of experimental parameters may allow a closer approximation to the expectations established from in vivo studies. The system developed sets the stage to further our understanding of signal transduction in synapsis by assigning specific functions to specific $A C h R$ subunits. The strategies described for AChR may be, in the near future, extended to other excitable channels, such as the $\mathrm{Na}^{+}$channel (Sigworth \& Neher, I980), the $\mathrm{K}^{+}$channel (Conti $\&$ Neher, I980) or the glutamate receptor (Patlak, Gration \& Usherwood, 1979). 


\section{(C) Porin}

(i) Background

Gram-negative bacteria have a multilamellar envelope consisting of a cytoplasmic membrane, a rigid peptidoglycan layer, and an outer membrane. The latter is composed of phospholipids, glycolipids, and proteins. One abundant protein of outer membranes is the so-called matrix protein, which in Escherichia coli, has a molecular weight of $3^{6} 500$. Porins, of which matrix protein is the only representative in $E$. coli $B^{E}$, are expected to comprise a major fraction of the proteins in the outer membrane of any Gram-negative bacterium (for a review see Nikaido \& Nakae, r979). Matrix protein spans the outer membrane and appears to interact with the peptidoglycan layer. Dodecyl-sulphatesolubilized matrix protein appears on electron micrographs as a hexagonal lattice with a unit cell composed of three units. It exhibits a 3-fold symmetry and triplet indentations penetrated by stain. These indentations were tentatively interpreted as membrane channels (Steven et al. 1977). Evidence that matrix protein forms membranespanning pores was inferred from the higher permeability of wild type Salmonella typhimurium to the antibiotic cephaloridine compared to a mutant deficient in porin (Nikaido et al. 1977).

\section{(ii) Vesicles}

Further support to this notion was obtained from reincorporation of isolated porin into phospholipid vesicles. These vesicles were permeable to hydrophilic molecules up to a mass of about 600 daltons (Nakae, I $976 a, b)$. It was suggested that porins were involved in the formation of large unspecific pores with the putative function of molecular sieves for the transmembrane diffusion of small hydrophilic solutes, such as sugars, amino acids and inorganic salts.

\section{(iii) Planar bilayers}

These findings prompted reconstitution studies in two laboratories using two different techniques of protein incorporation in planar bilayers (strategies 2 and 4 in Fig. 2). The starting material was matrix protein in an aggregated state, extracted from Escherichia coli envelopes (Rosenbusch, 1974). Schindler \& Rosenbusch (1978) formed planar membranes from proteoliposomes via spontaneous formation of 
monolayers and the subsequent apposition of two such monolayers to form a bilayer (see strategy 2 in Fig. 2). Conductance increases in large steps were observed at initial applied high membrane potentials (about $200 \mathrm{mV}$ ). This conductance initiation was irreversible and completed after several minutes. The activated conductance decayed in small regular conductance steps towards a steady-state level. The steps were assigned to the opening and closing of membrane channels with an open state conductance of $140 \mathrm{pS}$ in $0.1 \mathrm{M}-\mathrm{NaCl}$. Three salient features were analysed. (I) The voltage dependence of the distribution of channels in open or closed state. At sufficiently high voltages all channels closed. Upon voltage removal they opened again. Intermediate membrane potentials resulted in steady-state values of the open-closed distribution. As a direct consequence of this channel closing by voltage the steadystate relation between current and voltage showed a region of negative resistance. (2) The open-closed transitions at steady state resulted in a smaller deviation from the steady-state conductance level than expected from independent opening and closing of channels. This indicated that the open-closed transitions of the channels occur cooperatively. This result together with the apparent simultaneous activation of three or more channels provided evidence that the channels occur in clusters and that the clustered channels undergo cooperative open-closed transitions induced by voltage. (3) Channel triplets were observed at the lowest level of conductance activation. Triplets also exhibited reversible voltage-induced closing of the three channels in triplet. Finally, it was pointed out (Schindler \& Rosenbusch, 1978) that channel clusters and channel triplets correlate well with the ultrastructural analysis (Steven et al. 1977) of the arrangement of matrix protein in the bacterial cell envelope exhibiting lattices with three-fold symmetry as well as triplet indentations.

Benz et al. (1978) used strategy 4 in Fig. 2 as incorporation technique: Lipid bilayers were preformed and matrix protein was added to the aqueous phase containing detergent (sodium dodecyl sulphate or cholate at $\mathrm{I} \mu \mathrm{g} / \mathrm{ml}$ ). After addition of matrix protein to a final concentration of $0.5 \mu \mathrm{g} / \mathrm{ml}$ a continuous series of conductance increments was observed. Evidence for reaching a steady-state conductance was not presented. These increments attributed to channel-opening events were not uniform in size. The average value of the channel conductance, however, was comparable to that found by Schindler \& Rosenbusch (1978). In both studies the equivalent channel diameter was estimated to 
be about $\mathrm{I} \mathrm{nm}$ and the open-channel conductance was determined to be independent of voltage. However, in contrast to the results obtained by Schindler \& Rosenbusch, the occurrence of channels was spontaneous and the channels did not close with increased membrane potential (Benz et al. 1978).

Since then, more insight into channel formation by matrix protein has been obtained providing reasonable explanations to the initial disagreement. The initial report by Schindler and Rosenbusch was extended in two directions: Escherichia coli outer membrane vesicles and reconstituted vesicles containing single matrix protein trimers were transformed to planar membranes (Schindler \& Rosenbusch, 1980). The use of native outer membrane vesicles aimed to evaluate the physiological significance of the earlier results with isolated matrix protein aggregates and to gain insight into the properties which the channels may exhibit in an environment similar to that of the cell. It was found that planar membranes obtained from outer membrane vesicles showed similar electrical properties to those exhibited by matrix protein aggregates. Conductance activation at high voltage and the apparent cooperativity of channel state transitions indicated channel clustering. The observed channels could be reversibly closed and opened depending on the value of the applied voltage. The relaxation times for channel closing or opening exhibited a pronounced maximum at a voltage close to the midpoint of the negative resistance region in the current-voltage curve. Furthermore, voltage reversal experiments indicated that two closed-channel configurations exist for the two polarities of the applied voltage and that transitions between the two closed states occur via the open state. One additional feature was emphasized in view of the unknown function of matrix protein, or generally of porins, in bacterial outer membranes. With increasing membrane potential larger channel clusters were activated. All electrical characteristics of channels in large clusters (more than 30 channels) were distinctly different from those in smaller clusters. From small to large channel clusters, the cluster activation voltage significantly increased, the current-voltage relation changed from reversibility to a pronounced hysteresis loop, the characteristic times for the co-operative transitions between channel states slowed down by about one order of magnitude and even the channel conductance drastically changed from 0.8 to $0.5 \mathrm{nS}$ (in $\mathrm{I} \mathrm{M}-\mathrm{NaCl}$ ). Hysteresis and higher relaxation times beyond a critical cluster size had been theoretically predicted for 
a two-dimensional system of cooperatively interacting elements (Stankowski \& Gruenewald, 1980). Based on this coupling between channel activity and cluster size a delicate balance of the outer membrane permeability is expected. Modulations of channel clustering by interactions with the substratum of the outer membrane, peptidoglycan, or by smaller effectors at both membrane surfaces may effectively regulate the number of open channels (Schindler \& Rosenbusch, 1980).

Further results related to the compositional and organizational requirements for the restoration of the above defined channel activity were described (Schindler \& Rosenbusch, 1980). Matrix protein trimers were extracted from cell envelopes by repeated exposure to $\beta$-octylglucoside and purified by centrifugation and gel filtration. Such trimers, reincorporated into vesicles which were subsequently transformed into planar bilayers, did not elicit stable channel conductance. However, if trimers containing substoichiometric amounts of tightly associated glycolipid (lipopolysaccharide) were used, the channel conductance exhibited all characteristics recorded with planar membranes from outer membrane vesicles including negative resistance, cooperativity and hysteresis loops. Moreover, recombination of lipopolysaccharide- and glycolipid-free matrix protein trimers, both inactive if used alone, resulted in restoration of channel activity. These results clearly show that channel activity is contingent upon the presence of glycolipids in addition to matrix protein trimers. This was further detailed by forming planar bilayers from vesicles at extremely low trimer concentrations ( 1 trimer with associated glycolipid per $2.5 \times 10^{4}$ vesicles). An initial random distribution of about 20 trimers was expected in the resulting bilayer. In the first 2 min no channel conductance was observed. The first stable conductance corresponded to the activation of a channel triplet ( 3 channels). The number of activated channels slowly increased over a period of about $\mathrm{I} h$, almost exclusively in triplet steps (or small multiples thereof). It was concluded that the matrix protein trimerglycolipid complexes irreversibly associated to clusters and that this was required for the formation of channels exhibiting the characteristics found in outer-membrane-derived planar bilayers. This also allowed to estimate a lower limit for the diffusion constant of trimers of $10^{-10} \mathrm{~cm}^{2} / \mathrm{s}$. From the recovery of channels in relation to the expected number of trimers it was concluded that each trimer contains three channels (Schindler \& Rosenbusch, 1980).

Schein et al. (1976) reported that Paramecium mitochondria were the 
source of a voltage-dependent anion channel. This channel may be evolutionarily related to porin as will be discussed. The initial method of incorporation of the extract into the membrane followed the principle outlined in Fig. 2 (strategy I A). Briefly, mitochondria were sonicated with a 25-fold excess of soybean phospholipids, lyophilized and resuspended in hexane. This suspension was spread at an air-water interface to generate monolayers which were subsequently apposed to assemble a planar bilayer. Such bilayers display an increase in membrane conductance membrane which is stepwise and is attributed to the formation of channels of uniform size. The single-channel conductance in $0 . \mathrm{I} \mathrm{M}-\mathrm{KCl}$ is $450 \mathrm{pS}$ and it is $4.5 \mathrm{nS}$ in $\mathrm{I} \cdot 0 \mathrm{M}-\mathrm{KCl}$ (Colombini, 1979). These channels conduct anions better than cations of comparable size and charge; they display a twofold selectivity for $\mathrm{Cl}-/ \mathrm{K}^{+}$(see Colombini, personal communication in White \& Miller, 1979). The mitochondrial anion channel is voltage-dependent: the transmembrane voltage is in the vicinity of zero and decreases as a steep function of both positive and negative voltage (Schein et al. 1976; Colombini, 1979). At the level of the single channels the voltage dependence is manifested by the displacement of the channels to the closed state when the transmembrane voltage is increased both in the positive and in the negative direction. This channel activity has been described for mitochondria from rat liver, Neurospora and yeast (Schein et al. 1976; Colombini, 1979).

In a second method (strategy 4, Fig. 2), a mitochondrial suspension was solubilized with $2 \%$ triton $\mathrm{X}-100$ and this extract added to the aqueous compartment of an unmodified phospholipid bilayer: the incorporation of channels as a function of time showed a stepwise increase in membrane conductance (Colombini, 1979). Both methods yield the same channel activity and it was, therefore, concluded that the presence of the detergent had no effect on the channel activity (Colombini, 1979). The triton-extracts have been derived from rat heart, beef heart, and Neurospora mitochondria and from all these sources the characteristics of the conductance and of the channels are the same (Colombini, 1979).

By fractionating the mitochondrial membranes into outer and inner membranes, Colombini (1979) has identified the location of the voltagedependent anion channel to the outer mitochondrial membrane. Furthermore, considering the large size (about I $_{5} \AA$ ), its location in the outer mitochondrial membrane and its presence in a wide variety of 
organisms, Colombini (1979) has suggested that this channel may be the permeability pathway of the outer mitochondrial membrane to small molecules. This voltage-dependent anion channel has not yet been purified or characterized to a great extent. The activity in the bilayer has similarities to a recently reported porin from mitochondrial outer membranes (Zalman, Nikaido \& Kagawa, I980). Fragments of mitochondrial outer membrane obtained from rat liver or mung-bean seedlings were sonicated together with soybean phospholipids. The mixture was then dried under nitrogen and thereafter resuspended in an aqueous solution containing radioactively labelled markers. The assay consisted in measuring the exclusion limit of the permeability pathway to radioactively labelled oligo- and polysaccharides of various sizes: the exclusion limit lies between 2000 and 8000 daltons and most likely around $4000-6000$ daltons. Furthermore, by using this reconstitution assay, the channel-forming protein in the mung-bean mitochondria was identified as a protein or proteins with an apparent molecular weight of 30000 through differential detergent extraction in triton X-100 as well as with centrifugation of the extracted material in a sucrose density gradient (Zalman et al. 1980). The mitochondrial outer membrane porin was compared to the porin obtained from bacterial outer membranes. In all the bacterial strains so far examined porins are major outer membrane proteins with molecular weights in the range of $30000-$ 40000. In $E$. coli and $S$. typhimurium the exclusion limits of porin channels are around 600 daltons (Nakae, 1976). However, that of the porins from Pseudomonas aeruginosa outer membrane is similar to that of the mitochondrial outer membrane porin, that is, in the range of 4000-6000 daltons (Hancock, Decad \& Nikaido, 1979). In contrast, bacterial porin channels appear to be cation selective (Benz, Janko \& Läuger, 1979) whereas the mitochondrial outer membrane porin appear to be anion selective (Schein et al. 1976). Nevertheless both kinds of porin channels are voltage-dependent in the same direction; that is, the membrane potential closes the channel (Schein et al. 1976; Schindler \& Rosenbush, 1978).

Recently, Colombini (1980) reported a voltage-dependent anion channel from triton X-100 extracts of Neurospora crassa mitochondria. He estimated the pore size by measuring the permeability to nonelectrolytes of multilamellar liposomes generated from mixtures of phospholipids and $N$. crassa mitochondrial membrane material. $\mathrm{He}$ found that polyethyleneglycol of 3400 molecular weight was permeable 
while that of 6800 molecular weight was not. This provides an estimated diameter for the channels of $40 \AA$.

\section{(D) The proton channel of the proton-translocating ATPase}

(i) Background

The proton-translocating ATPase complex is ubiquitous throughout nature. It was first isolated and purified from the inner mitrochondrial membrane, later on from the thylakoid membrane of plant chloroplast, the plasma membranes of mesophilic and from thermophilic bacteria (for a review see Kagawa et al. 1979). Its structure when isolated from these very different sources appears to be remarkably similar. The $F_{1}$ ATPase is the catalytic moiety and the $F_{0}$ is the proton channel. In native membranes the ATPase complex operates as an ATP synthetase when a proton gradient exists across the membrane or as a proton pump when ATP is present as the substrate. Thermophilic bacteria have been extremely useful in the structure-function analysis of the protontransporting ATPase (Sone et al. 1979); the catalytic moiety $F_{1}$ has been crystallized; the complete reconstitution of $F_{1}$ from its five polypeptide subunits and demonstration of the function of the individual subunits in the hydrolysis of ATP as well as in the protontranslocation functions of each one of the subunits have been demonstrated.

\section{(ii) Vesicles}

Studies using the purified $F_{0}$ have indicated that it operates as a proton channel across membranes. $F_{0}$ is composed of at least three different subunits which are extremely hydrophobic. One of these subunits is a polypeptide of about 8000 daltons in molecular weight which probably exists as a hexamer (Sebald \& Wachter, 1978) and which binds DCCD (dicyclohexylcarbodiimide), a potent inhibitor of the membranebound ATPase (Cattel et al. 1970).

The amino acid sequence has been determined for the DCCD. binding protein of $F_{0}$ form Neurospora crassa, Saccharomyses cerevisiae, and $E$. coli (Sebald \& Wachter, 1977; Sebald, Machleidt \& Wachter, I980). The sequence is remarkably similar in the three preparations. The primary structure shows the existence of a short hydrophilic amino acid sequence in the centre of the protein, surrounded by two very hydrophobic segments of about 25 residues each. Close to the 
center of the hydrophobic chain that is located nearest the C-terminus there is an acidic side chain which reacts with DCCD. Sebald \& Wachter (1978) postulate that the two hydrophobic sections span the lipid bilayer and that the polar regions are in contact with the aqueous phase and the others subunits of the $F_{1}-F_{0}$ complex. It has been suggested (Sebald, Graf \& Lukens, 1979) that the DCCD-binding polypeptide probably assembles as a hexamer to provide a protonconductive pathway across the membrane.

This subunit has been purified from chloroplast by butanol extraction and reconstituted into phospholipid vesicles together with bacteriorhodopsin (Nelson et al. 1977). On illumination, no proton uptake could be measured unless DCCD was supplemented into the system. This indicates that the proton gradient generated by bacteriorhodopsin was immediately collapsed by the presence of the chloroplast proton channel and that this proton conduction ability could be blocked by addition of the inhibitor DCCD (Nelson et al. 1977).

When purified thermophilic $F_{0}\left(T F_{0}\right)$ was incorporated into lipid vesicles loaded with potassium, the addition of valinomycin caused a rapid uptake of protons through $T F_{0}$ (Okamoto et al. 1977). Both the rate as well as extent of proton translocation were greatly enhanced by increasing the amount of $T F_{0}$ reconstituted into the lipid vesicles. The proton channel reconstituted from thermophilic bacteria is not active unless all the three components of $T F_{0}$ are present. Similar results have been obtained from the $\mathrm{H}^{+}$-ATPase of Escherichia coli (Fillingame, 1976).

\section{(iii) Planar bilayers}

A butanol-soluble DCCD-binding proteolipid (or subunit 9) (Sebald et al. 1979, 1980) from yeast mitochondria was incorporated in planar bilayers using strategy $2 \mathrm{~B}$ of Fig. 2 (Schindler \& Nelson, unpublished results, I980). Butanol extracts from $\left[{ }^{35} \mathrm{~S}\right]$ methionine labelled mitochondria showed a single radioactive band that matched with the $\left[{ }^{14} \mathrm{C}\right] \mathrm{DCCD}-$ labelled proteolipid. The butanol extract was supplemented with an excess of soybean phospholipids ( $\mathrm{IO}^{5} \mathrm{~mole} / \mathrm{mole}$ ), dried into a thin surface film and, thereafter, suspended into vesicles at different $\mathrm{pHs}$ and $\mathrm{KCl}$ concentrations. Planar bilayers generated from these vesicle suspensions exhibited conductances in the range of $0^{\circ} \mathrm{I}-\mathrm{I} \mathrm{nS}$, at $\mathrm{pH}_{4} \cdot 0$. A $\mathrm{H}^{+} / \mathrm{K}^{+}$selectivity ratio of $800-2500$ was determined from Nernst potentials due to both $\mathrm{pH}$ and $\mathrm{KCl}$ gradients. 
This high selectivity for proton translocation was completely lost if chloroform or methanol was added to the solutions $(\sim 0.4 \%)$. The conductance was DCCD $\left(\mathrm{IO}^{-6} \mathrm{M}\right)$ sensitive: first it increased but then relaxed to less than $\mathrm{I} \%$ of the initial value within $10 \mathrm{~min}$. At high proton concentrations $(\mathrm{pH} \cdot 2)$ and with vesicles prepared from ether lipids and subunit 9, single channels of $18 \mathrm{pS}$ (at $100 \mathrm{mV}$ ) were clearly resolved. The channels opened and closed randomly with a mean open time of $\sim 5 \mathrm{~s}$. The single-channel conductance was independent of $\mathrm{KCl}$ concentration between $\mathrm{ro} \mathrm{mM}$ and $\mathrm{I} \mathrm{M}$. The conductance increased in a nonlinear fashion with increasing subunit 9 concentration in the vesicles. The molecularity of the channel has not yet been evaluated.

\section{(III) PuMPs}

Biological membranes reversibly transform several types of energy. One of the mechanisms that is prevalent in biological systems is the generation of electrochemical gradients across the membrane. The phenomenologic nature of this process has led to the concept of pumps. There is ample evidence indicating that the chemical energy of ATP is transformed into the electrochemical energy of an ion gradient by membranous ATPases. Efforts to incorporate the sodium-potassium ATPase in planar lipid bilayers have not led to satisfactory reconstitution. The results were recently reviewed by Shamoo \& Murphy (1979). We now turn to describe results obtained in planar lipid bilayers with two redox-driven pumps, namely the reaction centers (RCs) of photosynthetic bacteria and the mitochondrial cytochrome $c$ oxidase, as well as with the light-driven proton-pump, bacteriorhodopsin from Halobacterium halobium.

\section{(A) Reaction centres from photosynthetic bacteria}

(i) Background

The primary event in photosynthesis occurs in membrane-bound chlorophyll protein complexes known as reaction centres. In the primary event, light energy is used to transfer an electron from a donor (D) to an acceptor (A), thereby creating a charge separation against a redox potential difference. The chemiosmotic hypothesis of Mitchell (1968) postulates a light-induced electron transfer reaction that generates both a redox gradient and an electric field across the membrane. Therefore, the reaction centres, when incorporated and properly 
orientated in a planar lipid bilayer, may constitute the minimum photosynthetic entity that can generate a light-induced electric field. The use of planar bilayers affords one the possibility of studying the photochemical reactions by direct electrical measurements. Furthermore, since the reaction centres from photosynthetic bacteria are characterized to a great extent, this preparation offers the opportunity to correlate structural and photochemical studies of the isolated protein with the details of the photochemical reactions occurring across a membrane as detected electrically in the reconstituted planar bilayer.

The reaction centres from Rhodopseudomonas sphaeroides $\mathrm{R}-26$ are currently the best characterized (Feher \& Okamura, 1978). The protein component of the reaction centers is composed of 3 subunits present in equimolar proportions. They are called $\mathrm{L}, \mathrm{M}$, and $\mathrm{H}$ and they stand for light, medium, and heavy according to their molecular weight. Their combined molecular weight is at present estimated to be around 80000 daltons. The amino acid composition of the 3 subunits has been obtained and the amino acid sequence of the protein is under current investigation. Two of the subunits $(\mathrm{L}, \mathrm{M})$ contain all the pigments and primary reactants and can perform the primary photochemistry at low temperatures. However, the kinetics of the back reaction at low temperature (charge recombination) is different from that in chromatophores and from reaction centres that contain all the three subunits. The reaction centers contain four bacteriochlorophyll molecules and two bacteriopheophytin. Two bacteriochlorophylls constitute a specialized dimer that functions as a primary donor and bacteriopheophytin has been postulated to function as an intermediate acceptor. The spatial arrangement of the pigments has not been yet determined. In addition to the pigments, the reaction centres contain two ubiquinones and one iron molecule.

It is currently considered that in vivo, the water-soluble cytochrome $c_{2}$ serves as an exogenous electron donor to the reaction center. The cytochrome $c_{2}$ is located on one side of the membrane. The electron is transferred into the reaction centre and subsequently, is used to reduce a primary and a secondary ubiquinone. The kinetics of the different steps in this photochemical reaction have been well characterized in detergent-solubilized reaction centers. The transmembrane events associated to these photochemical reactions cannot be studied in detergent solutions. Furthermore, the electrogenic nature of the primary photoinduced charge separation has been demonstrated with a 
variety of indirect methods applied to the native chromatophore membrane.

\section{(ii) Large vesicles}

The reaction center-lipid complex in hexane (see I, C (i), p. I5; Schönfeld et al. 1980) can be used to generate large unilamellar vesicles containing the reaction centres by the procedure indicated in Fig. 2 (strategy $2 \mathrm{~B}$ ) (Darszon et al. 1980). Freeze-fracture replicas of the protein vesicles containing reaction centres reveal that both internal and external leaflets contain numerous intramembranous particles of about roo $\AA$ in diameter with some rod-shaped particles that were about $200 \AA$ long. The internal aqueous space of the reaction center-lipid vesicles was about $13 \mu \mathrm{l} / \mathrm{mg}$ of lipid. Absorbance spectra of the reaction center incorporated in the large vesicles indicated a preservation of both the spectral integrity and the activity of the protein. The dark spectrum exhibited the three peaks in the infrared region at 760,802 , and $865 \mathrm{~nm}$, which are characteristic of purified reaction centers (Feher \& Okamura, 1978). The most prominent light-induced absorbance change in the spectrum is the bleaching at $865 \mathrm{~nm}$ that accompanies the primary photochemical reaction; this was indeed observed in the large vesicles. The reaction centers incorporated into the large lipid vesicles were shown to translocate protons across the membrane. A vesicle suspension was supplemented with reduced cytochrome as well as with ubiquinone- $O$ and a lipid-soluble hydrogen atom carrier, diaminodurene $(2,3,5,6$-tetramethyl- $p$-phenylenediamine), which probably mediates the closing of the redox cycle and the actual transfer of protons out of the vesicles. On illumination, a rapid efflux of protons which attained a steady-state in about io $\mathrm{s}$ was recorded. When the light was turned off, the $\mathrm{pH}$ resumed its baseline value with a decay time constant that was primarily determined by the intrinsic proton permeability of the vesicle membrane. Addition of a proton ionophore-fluorocarbonylcyanidephenylhydrazone (FCCP) collapsed the light-induced $\mathrm{pH}$ gradient and no net accumulation of protons was recorded. These results are in agreement with reported values for reconstituted sonicated vesicles containing reaction centers (Crofts et al. 1977).

A possible mechanism for the proton translocation reaction considers that reduced cytochrome $c$ located on the outside of the vesicles donates electrons to the reaction centres incorporated in the membrane. On 
illumination, there is a transmembrane electron transfer reaction that results in the reduction of coenzyme $Q$ located on the inside of the vesicles which together with two protons derived from the aqueous internal space result in the formation of reduced coenzyme $Q$. The latter reduces diaminodurene, which thereby shuttles to the outside of the vesicles where it releases two hydrogen atoms: two protons to the aqueous solution and two electrons. Diaminodurene in the oxidized form then returns to the inside of the vesicles where it can be subsequently reduced by the photogenerated reduced quinone. Thus, the actual transfer of protons out of the vesicles and the closing of the redox cycle is mediated by diaminodurene. This mechanism was previously postulated for the effect of phenazinemethosulphate or tetramethylparaphenyelenediamine in chromatophore membranes (cf. Skulachev, 1975). These results demonstrate that the reaction centre can be incorporated into large lipid vesicles of several microns in diameter and display light-induced proton translocation.

\section{(iii) Planar bilayers}

The more direct approach of incorporating the reaction centers into lipid bilayers in order to measure the transmembrane events was initiated by Drachev et al. (1975, 1976a). Reaction centers and soybean phospholipids were mixed and sonicated in detergent, followed by dialysis removal of the detergent. The vesicles formed were allowed to fuse with black lipid films or even with thick lipid films of thickness greater than $1000 \AA$ in the presence of $\mathrm{Ca}^{2+}$. On illumination, a potential difference developed, the sign of the potential being negative on the compartment where the vesicles were initially added; the potential vanished in the dark. The extent of the light-induced membrane potential increased in the presence of a lipid-soluble hydrogen atom carrier and by including coenzyme Q6 in the sonication mixture. The photoeffect displayed an action spectrum similar to the reaction centre absorption spectrum and it was inhibited by $O$-phenantroline, an inhibitor of electron transfer. This investigation is subject to reservation since the structure of fused vesicles with thick films is not well understood and the nature of the reactions is not clear.

The well-characterized reaction centers were recently incorporated into planar lipid bilayers. Two methods outlined in Fig. 2 (strategies I $B$ and 2 A) were used. First, we will consider the preparation derived from a reaction center-lipid complex extracted in hexane (see I, C (i)). 
Planar bilayers were formed by apposing two reaction center-lipid monolayers assembled from the reaction center-lipid complex in hexane (strategy I B, Fig. 2). When supplemented with secondary donors, such as cytochrome $c$, and acceptors, such as ubiquinone- $O$, on opposite sides of the membrane the reconstituted bilayers generate photovoltages and photocurrents. The growth of the open-circuit photovoltage was biphasic having an initial fast component and a slower exponential growth heading towards a steady-state value. After the light was turned off the voltage decayed with a single exponential corresponding to the product of the resistance and capacitance of the system. The time course of the corresponding short-circuit photocurrent consists of an initial current spike and a steady-state current. When the white light was turned off a negative current transient was observed which quickly relaxed towards zero. The wavelength dependence of the photoresponse matched the absorption spectrum of the reaction centers and, therefore, unequivocally identifies the reaction centers as the molecular species responsible for the photoelectric effect. The effect of external components on the photocurrent was also studied: in the absence of cytochrome $c$ no photocurrent was detected. In contrast, if the exogenous $\mathrm{Q}-\mathrm{O}$ was deleted the initial transient was still present but the steady-state current was zero. Omission of the exogenous quinone Q-1o from the reaction center-lipid complex before the assembly of the monolayers eliminated the transient current but not the steady-state current. $O$-phenantroline, which inhibits electron transfer between the primary and secondary quinone, when added to the compartment containing Q-O abolished the entire photoresponse. Similar results were obtained when the monolayers were derived from reaction centers incorporated into lipid vesicles (strategy $2 \mathrm{~A}$, Fig. 2). The vesicles containing the reaction centres were assayed in advance for their competence in the primary photochemistry (Schönfeld et al. I980, unpublished results).

In an independent study (Packham et al. 1980) reported similar results obtained with a reaction center-lipid complex extracted into octane. The extraction procedure is a variation of the one described before with the use of $\mathrm{Ca}^{2+}$ instead of $\mathrm{Mg}^{2+}$ and octane instead of hexane. The reaction center-phospholipid complex in octane was used to prepare black lipid membranes or planar membranes with a thickness greater than $500 \AA$. There was no consistent difference in the photoresponses exhibited by the two kinds of planar membranes (Packham 
et al. 1980). The reaction centers in the phospholipid octane solution were shown to retain photochemical activity. The kinetics of the flashinduced reaction center bacteriochlorophyll dimer oxidation and its dark reduction were studied. In the absence of supplementary quinone the oxidation of the bacteriochlorophyll dimer was recorded followed by the subsequent reduction in the dark. The half-time of the dark process was about $75 \mathrm{~ms}$ which was similar to the half-time of return of an electron to the oxidized bacteriochlorophyll dimer from the primary endogenous quinone measured in detergent-solubilized reaction centers. It was, therefore, concluded that the secondary quinone was lost in the preparative procedures. Indeed, addition of ubiquinone-Io to the reaction center-phospholipid octane solution increased the amount of bacteriochlorophyll that could be oxidized in the millisecond time scale following the flash, and furthermore prolonged the time course of reduction in the dark, now to a half-time of ${ }_{1} 65 \mathrm{~ms}$. This retardation suggests that the added ubiquinone reconstituted the electron transfer reaction between the primary and secondary quinone. In agreement with this contention, addition of $O$-phenantroline accelerated the time course of dark re-reduction of the oxidized bacteriochlorophyll dimer. Planar membranes derived from the reaction center-phospholipid octane solution display no photoelectric responses unless ferrocytochrome $c$ is added to one side of the membrane. Furthermore, the addition of ubiquinone- 10 to the reaction center-phospholipid octane solution prior to bilayer formation increases the amplitude of the short-circuit photocurrent. This effect is in agreement with the photochemical results in which the excess ubiquinone increases the amount of photo-oxidizable bacteriochlorophyll by increasing the number of reaction centres which contain a full complement of ubiquinone. That this is indeed the case emerges from the fact that $O$-phenantroline removes the enhancing effect of supplementary quinone and reduces the magnitude of the photoresponses to that prevalent in the unsupplemented condition. The action spectrum of the peak current response generated during the steady-state illumination is in agreement with the absorption spectrum of the reaction center-phospholipid octane solution. All these results are in agreement with those obtained by Schönfeld et al. (1979).

Further results using a series of single turnover xenon flashes spaced $25 \mathrm{~ms}$ apart showed that the first flash yields only a small response while subsequent flashes elicit much larger current transients. However, if the bilayer is supplemented with ferricyanide on the side opposite to that 
where ferrocytochrome $c$ is added, the light-induced photocurrents are of the same amplitude including the first one (Packham et al. 1980). These results indicated that the symmetric planar membrane contains two reaction-center populations of opposite orientation; on the first turnover, both sets of reaction-center populations are activated but their opposing current transients effectively cancel. However, by the second turnover only the reaction-center population with an orientation accessible to reduced cytochrome $c$ is returned to a functional state while the other is still essentially in the photochemically inactive oxidized state. The addition of the membrane impermeant potassium ferricyanide to the side opposite to that containing reduced cytochrome $c$ chemically oxidizes only the reaction-center population inaccessible to reduced cytochrome $c$ and thereby allows a net current transient following the first turnover (Packham et al. 1980).

These experiments demonstrate that reaction centers incorporated into planar lipid bilayers generate photoelectric signals that arise from the transfer of electrons from a secondary donor to a secondary acceptor located on opposite sides of the membrane and, therefore, provide direct demonstration for the transduction of light energy into a transmembrane electric current. Furthermore, the reconstituted planar bilayer may favour the search for the function of the individual subunits of the reaction centers which so far has remained elusive. In addition, the system offers the possibility of investigating the details of the interactions between secondary reactants and reaction centers and the coupling between redox potentials and membrane potentials.

Because the reaction center-lipid complex in hexane is the starting material for the formation of both the large vesicles (Darszon et al. 1980) and the planar lipid bilayers assembled from monolayers (Schönfeld et al. 1979), the methods may be used in conjunction to complement each other; the vesicle system allows a more direct correlation of membrane composition parameters, such as protein concentration, with functional parameters. Furthermore, the large size allows entrapment of larger markers as well as enables the study of the electrical properties with microelectrode or other techniques. We can foresee that the combination of these two reconstituted reaction center-lipid bilayer systems will enhance our understanding of the mechanism of lightinduced transmembrane electron transfer and its role in phototransduction. 
(B) Cytochrome c oxidase

\section{(i) Background}

Cytochrome $c$ oxidase is a mitochondrial inner membrane enzyme which catalyses the final reaction of respiration by reducing molecular oxygen to water with the electrons of the respiratory chain funnelled through its substrate, cytochrome $c$. There is considerable evidence indicating that the enzyme spans the entire width of the inner mitochondrial membrane, interacting with cytochrome $c$ on the cristae side, and with oxygen on the matrix side (cf. Lemberg, 1969; Prochaska, Bisson \& Capaldi, 1980). This electron transfer reaction in mitochondria is associated with the synthesis of one molecule of ATP. Such a transmembrane orientation is a requirement in the chemiosmotic hypothesis for energy coupling (Mitchell, 1968) in which the orientated protein allows electrons to flow across the mitochondrial membrane in a particular direction. As a consequence of this vectorial movement of electrons, charge separation occurs across the membrane; electrons on one side, protons on the other. Accordingly, such a transmembrane redox reaction can be thought of as an electrogenic proton pump. The energy of the resulting proton gradient, Mitchell postulates, is transformed into ATP by a reversible proton-translocating membrane-bound ATPase (Mitchell, 1968). The basic concept of transmembrane electron transfer is analogous to that described for the bacterial reaction centres.

Cytochrome $c$ oxidase is a membrane protein composed of, apparently, seven polypeptide subunits. Three of these subunits are synthesized by the mitochondrial genome. In addition, it contains two copper atoms and two haems. All the haem and copper are located in subunits I and II. Subunit II appears to be the binding site for cytochrome $c$. Subunits II and III are exposed to both sides of the membrane (Prochaska et al. I980).

A great deal of progress is rapidly emerging in the studies of the structure of cytochrome $c$ oxidase. Vesicles formed from a triton preparation stained with uranyl acetate show oriented dimers whereas the deoxycholate-derived preparation gives a two-dimensional array of unorientated monomers (Henderson, Capaldi \& Leigh, 1978; Fuller, Capaldi \& Henderson, 1979). Furthermore, recently three-dimensional crystals were generated from octylglucoside-extracted cytochrome $c$ oxidase by ammonium sulphate precipitation techniques, these may become useful for X-ray diffraction studies (Capaldi, personal com- 
munication, I980). In addition, a complex of cytochrome oxidase and cytochrome $c$ has been crystallized in the form of needles (Ozawa, Suzuki \& Tanaka, 1980). Moreover, the amino acid sequence of all the subunits can be obtained through DNA sequencing techniques (Thalenfeld \& Tzagoloff, 1980; Barrell, Bankier \& Dvouin, 1979). It appears, therefore, that cytochrome oxidase may become the first multisubunit membrane protein to be understood at the molecular level.

\section{(ii) Vesicles}

Cytochrome $c$ oxidase was incorporated into vesicles (Hinkle et al. 1972; Jasaitis et al. 1972). Such reconstituted cytochrome oxidase vesicles catalyse the oxidation of external reduced cytochrome $c$ and the velocity of oxidation is greatly stimulated by addition of proton conductors, or by breaking the membrane with detergents (Skulachev, 1975; Hinkle, 1973). This experiment suggested that the oxidation rate in the cytochrome oxidase vesicle was modulated by the membrane potential, a phenomenon reminiscent to the so-called respiratory control in intact mitochondria.

Recent functional studies with cytochrome oxidase have provided evidence consistent with the notion that cytochrome oxidase is a redoxlinked proton pump. (Wikstrom \& Krab, I979). This concept is fundamentally different from the electron translocating function postulated by Mitchell (I968) and described before. The proton pumping model of cytochrome oxidase considers that the electron donated by cytochrome $c$ to cytochrome oxidase is not transferred across the membrane in order to reduce oxygen but is donated on the same side of the membrane where cytochrome $c$ is located. The reaction mechanism requires the presence of protons in order to form water, therefore, it is postulated that two protons are transported across the membrane from the opposite side to the cytochrome $c$. This scheme yields a proton translocated to electron-consumed ratio $\left(\mathrm{H}^{+} / \mathrm{e}^{-}\right)$of one. Purified cytochrome oxidase was incorporated into lipid vesicles and used to determine the occurrence of a redox-linked proton ejection from the vesicles. When a suspension of cytochrome oxidase vesicles was reduced with ferrocytochrome $c$, a fast efflux of protons was observed in the medium followed by net proton uptake. This experiment was performed in the presence of potassium and valinomycin to allow for the fast charge compensation. The fast proton efflux was fully abolished by 
proton ionophores or by omitting valinomycin from the medium (Krab \& Wikstrom, 1978). Casey, Chappell \& Azzi (1979) confirmed these results with cytochrome oxidase vesicles. Furthermore, by careful titration with the proton ionophore FCCP they showed that proton ejection was indeed the result of proton translocation and reported a $\left(\mathrm{H}^{+} / \mathrm{e}^{-}\right)$ratio of 0.9 . Casey, Thelen \& Azzi (1980), showed that DCCD bound to the reconstituted oxidase in a time- and concentrationdependent profile that correlated with the DCCD-induced inhibition of $\mathrm{H}^{+}$-translocation.

These new results on the proton translocation ability of cytochrome oxidase in reconstituted vesicles indicate that a re-evaluation of the electron translocation concept for cytochrome oxidase is necessary. Furthermore, the discovery that subunit III of cytochrome oxidase exhibits the binding site for DCCD (Capaldi, personal communication) with the same amino acid sequence at the binding site of the proton channel of the proton translocating ATPase suggests that this subunit may also have a proton channel function for cytochrome oxidase. This suggestion has some experimental support in the fact that purified cytochrome oxidase from Paracoccus dentifricans (Ludwig \& Schatz, 1980) which only contains two subunits and lacks subunit III of cytochrome oxidase, when incorporated into vesicles exhibits a $\left(\mathrm{H}^{+} / \mathrm{e}^{-}\right)$ of less than $0 \cdot 2$. It would be interesting to supplement this cytochrome oxidase with purified subunit III from beef heart mitochondria and determine the increase in the $\left(\mathrm{H}^{+} / \mathrm{e}^{-}\right)$. If this were possible the purification of subunit III would allow the direct assessment of its proton channel activity by incorporating the purified subunit III into planar lipid bilayers and directly recording the proton channel conductance. This may also allow the evaluation of whether the channel is formed by one subunit or through the formation of hexamers as postulated by Sebald \& Wachter (1978). Such strategy has the advantage that the inhibitor DCCD can be used to establish the specificity of the proteininduced conductance. Unpublished results by Capaldi have indicated that DCCD can inhibit up to $80 \%$ of the proton-pumping ability of cytochrome oxidase and up to $60 \%$ of its redox ability, thus suggesting that indeed subunit III, the putative proton channel, may be actually involved in the reaction mechanism (Capaldi, personal communication, 1980). 
(iii) Planar bilayers

Cytochrome oxidase vesicles adsorbed or fused on to black films or thick films generated cyanide-sensitive transmembrane potential upon oxidation of external cytochrome $c$. This system has not been further characterized (Drachev et al. 1974).

Cytochrome oxidase was incorporated into planar lipid bilayers according to a protocol outlined in Fig. 2 (strategy I B) (Montal, 1974). Cytochrome oxidase was incorporated into lipid vesicles by the detergent dialysis technique. After addition of $\mathrm{Ca}^{2+}$, a virtually complete extraction (over $90 \%$ ) of the cytochrome oxidase-lipid complex into $n$-hexane occurred. No extraction was obtained in the absence of $\mathrm{Ca}^{2+}$. The activity of the extracted enzyme was assayed by removing the hexane by solvent evaporation and subsequent hydration of the residue in salt media. The enzymic specific activity of cytochrome oxidase was comparable to that of the original detergent extract; the first-order character of the reaction and its reactivity to cyanide were preserved. However, this strategy of assembly of biologically active planar bilayers requires the spreading of the protein lipid extract at an air-water interface (Montal, 1974). Since cytochrome oxidase must remain native after spreading an assay at this stage of the process is necessary. The surface activity of the cytochrome oxidase-lipid complex was compared to that of the equivalent phospholipid. The cytochrome oxidase lipid-complex exhibited an expanded surface pressure $v$. area per molecule behaviour with an area per phospholipid molecule of $8_{3} \AA^{2}$ at collapse pressure. In contrast, soybean lecithin was compressed monotonically with an area per phospholipid molecule of $6 \mathrm{I} \AA^{2}$ at collapse pressure. Furthermore, cytochrome $c$ oxidase-lipid complex spread at an air-water interface over a surface containing reduced cytochrome $c$ catalysed the oxidation cytochrome $c$. The reaction, monitored spectrometrically, showed firstorder kinetics and was cyanide-sensitive. The oxidation of cytochrome $c$ was indeed the result of enzymic activity of the monolayer since increasing the amount of protein in the monolayer, at first, resulted in an enhancement of oxidation but as the amount of cytochrome oxidase was increased further its specific activity decreased reaching the maximum at the concentration close to that at which collapse of the monolayer occurred. Moreover, the first-order rate constant reached a plateau, indicating that when all the surface is covered by the monolayer the reaction proceeded at maximum velocity. This was taken as evidence 
that the cytochrome oxidase complex retained its enzymic activity after being spread at an interface.

Planar lipid bilayers containing cytochrome oxidase were formed by apposing two cytochrome oxidase-lipid monolayers. Such bilayers formed in the presence of oxidized cytochrome $c$ in one compartment established a membrane potential upon reduction of the substrate; this was prevented or reversed by cyanide. Ascorbate and cyanide per se did not modify the conductance of the bilayers. The potential was not generated if cytochrome $c$ was absent or when the membranes were formed with heat-denatured enzyme. The origin of the potential difference was attributed to the transmembrane electron transfer reaction (Montal, 1974). These results were essentially confirmed by Chien \& Mueller (1976), who extracted cytochrome oxidase with phospholipids into octane or decane and formed black lipid membranes from the cytochrome oxidase-lipid solution in solvents. A study on the incorporation of cytochrome oxidase into a new form of planar synthetic membrane was reported by Tredgold \& Elgamal (1979). The nature of the new method of membrane formation and the lack of sufficient documentation of the results make evaluation of this report difficult at the present time. Considering the importance of the new results related to proton pumping by the oxidase one should await experimental verification before accepting the electron-translocating or protontranslocating model for the cytochrome oxidase, and in this respect the use of planar lipid bilayers may provide the direct answers.

\section{(C) Bacteriorhodopsin}

(i) Background

Bacteriorhodopsin is a retinal-containing membrane protein that resembles the visual pigment rhodopsin in chemical composition and photochemistry. It is the only protein component in the purple membrane of Halobacterium halobium (for recent reviews see Henderson, 1977; Korenbrot, 1977; Stoeckenius, I980). Stoeckenius and coworkers have shown that bacteriorhodopsin is a light-driven proton pump; it moves protons from the intracellular space to the external medium. The resulting proton gradient is then utilized for ATP synthesis, in accordance with Mitchell's hypothesis of energy coupling (rg68). Thus bacteriorhodopsin functions as a photon energy converter similar to photosynthetic membranes. 
Bacteriorhodopsin comprises about $75 \%$ of the dry weight of the purple membrane, has a molecular weight of about 25000 and is a single polypeptide chain. Recently, three-dimensional crystals of bacteriorhodopsin have been generated which may be suitable for X-ray diffraction studies (Michel \& Oesterhelt, 1980). Furthermore, the complete amino acid sequence of the bacteriorhodopsin has been obtained and the possible array of the polypeptide chain within the lipid bilayer has been proposed (Ovchinikov et al. 1979). Therefore, bacteriorhodopsin appears to provide a unique prototype for the eventual structural and functional understanding of a membrane protein.

Bacteriorhodopsin has a retinal chromophore complexed to the protein by a protonated Schiff-base linkage. The chromophore is the alltrans isomer when the maximum absorption is at $570 \mathrm{~nm}$. Upon illumination, bacteriorhodopsin proceeds through a series of conformational transitions which have been characterized by flash spectroscopy and identified by their wavelength of maximum absorbance (Lozier, Bogomolni \& Stoeckenius, 1975). At least five spectroscopically distinct intermediates have been identified in this bleaching cycle. Associated to the photochemical changes there are proton translocation events whose kinetics have been measured with indicator dyes(Bogomolni et al. 1976,1980$)$.

\section{(ii) Vesicles and interfacial films}

The purple membrane fragments have been incorporated into vesicles by the detergent dialysis technique as well as by sonication (Racker $\&$ Stoeckenius, 1974, Racker, 1973). Proton uptake was measured when these vesicles were illuminated, as was proton release in the dark. Proton uptake was abolished by proton ionophores and the rate of proton uptake and release was accelerated by valinomycin in the presence of potassium. This provided evidence for the postulated vectorial transpert of protons.

The direct measurements of photocurrents or photovoltages in planar bilayers with incorporated bacteriorhodopsin has been a subject of considerable study. This is accounted for by the fact that flash spectroscopy provides information only on the absorption properties of the chromophore in the protein but the relationship between the single photochemical reaction steps and the proton transport process cannot be directly established. One approach to this question consists in 
orienting the purple membrane at polar-apolar interface and recording directly the photoelectric signals induced by photoexcitation. Interfacial films with orientated bacteriorhodopsin (over $85 \%$ ) have been formed on a water surface (Hwang et al. $1977 a, b$ ). These films contain spectroscopically intact bacteriorhodopsin and upon illumination they generate photopotentials. The interfacial films can be formed at the hydrocarbonwater interface and if the hydrocarbon (decane) is supplemented with a lipid-soluble proton carrier such as FCCP, the amplitude of the photopotential is considerably increased. The polarity of the photopotential indicated that in the light positive charges were transferred from the aqueous to the apolar phase through the interfacial film. This indicated that the orientated bacteriorhodopsin molecules were translocating protons from the aqueous to the non-aqueous phase which corresponds to the translocation of protons from the intracellular to the extracellular surface of the native bacteriorhodopsin-containing membrane. The interfacial films were generated by spreading onto an air-water interface a suspension of sonicated fragments of purple membrane in a solution of soybean lecithin in hexane prepared as outlined in Fig. 2 (strategy I A) (Hwang et al. 1977a,b).

In another set of studies interfacial films containing bacteriorhodopsin were generated by directly spreading purple membrane patches at an interface (strategy $2 \mathrm{D}$, Fig. 2). Thereafter, the film was overlaid with a thin layer of hexane and the surface film at the water-hexane interface was immediately apposed to a vertically mounted Teflon septum which separated to aqueous compartments (Trissl \& Montal, 1977; Hong \& Montal, 1979). This method assures that only capacitative photoresponses be detected since the $\mathrm{dc}$ resistance of the Teflon film is as high as $10^{14} \Omega$. Using a $\mathrm{I} \mathrm{ms}$ light flash, an open-circuit photovoltage was detected which raised in about $15 \mathrm{~ms}$ and decayed in about i s (Trissl \& Montal, 1977). If a $300 \mathrm{~ns}$ dye laser pulse was used the same time course was reproduced but a small positive signal with unresolved latency was discerned (Hong \& Montal, 1979). The corresponding short-circuit displacement photocurrent exhibited two transient peaks of opposite polarities. The initial phase has a positive polarity on the side where the bacteriorhodopsin layer was deposited. This component, $B_{1}$, of the displacement photocurrent has no detectable latency at an instrumental time constant of $\mathrm{I} \cdot 5 \mu \mathrm{s}$ and persists at $5{ }^{\circ} \mathrm{C}$. The second slower component, $B_{2}$, has an opposite polarity and relaxes with an exponential time constant of about $\mathrm{I} 60 \mu \mathrm{s}$. The $B_{2}$ component is 
inhibited by low temperature $\left(5^{\circ} \mathrm{C}\right)$ and low $\mathrm{pH}(\mathrm{pH}=3 \cdot 0$ ) (Hong \& Montal, 1979).

Using a I $_{5}$ ns laser pulse, Drachev, Kaulen \& Skulachev (1978) recorded several open-circuit displacement photovoltages in a bacteriorhodopsin model membrane. Although they did not identify them as capacitative signals the kinetic characteristics are similar to those recorded at the Teflon-water interface (Trissl \& Montal, 1977; Hong \& Montal, 1979). In the study of Drachev et al. (1978) the membranes were formed by associating bacteriorhodopsin-containing vesicles with phospholipid-impregnated membrane filters (Drachev et al. 1976b). Collodion films were used lately because they provide a higher electrical capacity (Drachev et al. 1978). The collodion film is impregnated with a decane solution of asolectin and then mounted as a septum in a two-compartment chamber. Thereafter, bacteriorhodopsin-containing vesicles were added to one of the two aqueous compartments and supplemented with $15-30 \mathrm{~mm}-\mathrm{CaCl}_{2}$. The mixtures were stirred overnight. The recorded signals consisted of an initial phase with a submicrosecond rise time and with the same polarity as the $B_{1}$ component of Hong \& Montal (I979); a second phase which rises in 20$50 \mu \mathrm{s}$ and with the same polarity as the $B_{2}$ component of Hong \& Montal (1979), a third component of the same polarity as the second one and with a rise time of 6-12 $\mathrm{ms}$ and a slow one with a relaxation time of I second were also recorded (Drachev et al. 1978). A tentative assignment of the relaxation kinetics of the photointermediates associated with the $B_{1}$ and $B_{2}$ components was considered. The $B_{1}$ component as well as the first phase of Drachev et al. (1978) may be generated by the formation and decay of the $\mathrm{K}_{590}$ intermediate (Hong \& Montal, 1979; Drachev et al. 1978; Stoeckenius, Lozier \& Bogomolni, 1979). The $B_{2}$ component and the second phase of Drachev et al. (1978) may be correlated with the formation and decay of $\mathrm{M}_{412}$. Keszthelyi \& Ormos (1980) measured simultaneously the electric-and light-absorption signals in suspensions of purple membrane orientated by an electric field. Their results provide direct evidence for the assignment of the source of the displacement signals outlined before. Hwang et al. (1978) extended these results using multilayers of dry purple membranes in lipids sandwiched between two metal electrodes; in addition, they recorded charge displacements associated with each of the photointermediates.

Korenbrot \& Hwang (1980) have recently reported the assembly of membranes by transferring a film containing non-overlapped orientated 
single-sheet fragments of purple membrane in lipid from the air-water interface onto a thin electrically conductive support case from nitrocellulose. The film under support is thereafter transferred through a second interface lipid film. Illumination produces photocurrents and photovoltages whose action spectra agree with the absorption spectrum of bacteriorhodopsin. For a step of light the waveform of the photocurrent consisted of a transient and a steady-state current; the amplitude of the photocurrent was proportional to membrane area and bacteriorhodopsin concentration. The photovoltage followed the current in amplitude and kinetics as expected from a simple resistance-capacitance network. Moreover, transient photovoltages without a detectable latency and which reach a peak in $100 \mu$ s were measured and interpreted as probably arising from intramolecular charge displacements.

Therefore, the capacitative photoresponses in the bacteriorhodopsin model membranes provide a new dimension in the study of the conformational dynamics of bacteriorhodopsin by following the charge displacements which accompany the photochemical transformation.

\section{(iii) Planar bilayers}

A number of recent studies have been performed using planar lipid bilayers. Hermann \& Rayfield (1978) prepared bacteriorhodopsincontaining vesicles by the sonication technique and incubated vesicle suspensions with black lipid membranes prepared from egg lecithin. The vesicle suspension was incubated with the black lipid membrane for 4-5 $\mathrm{h}$ and then removed by perfusion. After 'vesicle fusion' the surface of the black lipid membrane developed a silvery sheen suggesting an increase of membrane thickness. Danchazy \& Karvaly (1976), Danchazy et al. (1978) and Bamberg et al. (1979) used black lipid membranes prepared from I \% diphytanoyl lecithin and $0.025 \%$ octadecylamine in decane. The positively charged surfactant promoted the interaction of purple membrane fragments with the planar membrane yielding stable adsorption of membrane sheets into the surface of the black membrane. In both studies the light-induced short-circuit current exhibits a large displacement photocurrent when the light is turned on and a very small steady-state photocurrent. The latter increases considerably when the lipid bilayer is supplemented with proton conductors such as gramicidin or CCCP (Bamberg et al. 1979; Hermann \& Rayfield, 1978). The sign of the photocurrent was such that proton transfer occurred 
toward the bacteriorhodopsin-free compartment (Bamberg et al. 1979). The action spectrum of the stationary photocurrent (Bamberg et al. 1979) as well as of the transient photovoltage (Danchazy \& Karvaly, 1976) are in close agreement with the absorption spectrum of bacteriorhodopsin. Furthermore, Danchazy et al. (1978), investigated the origin of the photoelectric response by moving a small actinic light spot across the plateau-Gibbs border and the bilayer part of the planar membrane. The scanning experiments showed that the photoelectric signals were generated by the bacteriorhodopsin incorporated or adsorbed onto the bilayer part of the model membrane. The authors did not rule out the possibility that during attachment of bacteriorhodopsin to the bilayer the latter might have formed microlenses of a smaller size than the distance resolution of the scanning technique used.

The observation that the steady-state photocurrent strongly increases in the presence of either gramicidin A (Bamberg et al. 1979) or a proton conductor, CCCP (Hermann \& Rayfield, I978) argues against the contention that bacteriorhodopsin is actually incorporated into the black film and spans the width of the bilayer exposing polypeptide surfaces to both aqueous compartments. The most likely interpretation of these findings (Bamberg et al. 1979) assumes that purple membrane sheets are associated to the black lipid membrane in a preferential orientation. Since the proton permeability of the modified black film is low only the transient displacement photocurrent occurs; in contrast, in the presence of proton conductors, a steady-state photocurrent can flow between the two aqueous compartments. This model was analysed on the basis of an equivalent circuit (Hermann \& Rayfield, 1978; Bamberg et al. 1979) in which the current source is connected in parallel with the membrane resistance and membrane capacitance and considers that the current generator is linearly dependent on voltage. Bamberg et al. (1979) furthermore reported that when the retinal-free purple membrane (apomembrane) was attached to the lipid bilayer the photoresponse was marginal but could be restored by adding all-trans retinal to the aqueous phase suggesting that the apomembrane bound to the planar bilayer was reactivated in situ.

None of these studies deal with a genuine incorporation of bacteriorhodopsin into a planar lipid bilayer where bacteriorhodopsin regains a transmembrane orientation analogous to that existent in the bacterial membrane; it would, therefore, be desirable to show that oriented bacteriorhodopsin incorporated into planar lipid bilayers generate 
photoelectric signals that arise from the transfer of protons across the membrane.

\section{(IV) Perspectives in membrane reconstitution}

In light of this account it emerges that planar bilayers in conjunction with vesicles and monolayers constitute a powerful approach for investigating the mechanism of signal transduction at the molecular level. The strategies developed over the last io years for the reassembly of functional membrane proteins in planar bilayers are still in their infancy but may, in the near future, become routine techniques in membrane biology and may open new avenues to deepen our knowledge on the molecular basis of energy transduction and information processing by biological membranes.

\section{ACKNOWLEDGEMENTS}

This work was supported by grants from the National Institutes of Health (EY-02084) and the Office of Naval Research (Nooor4-79-C0798).

\section{(V) REFERENCES}

Adams, D. J., Dwyer, T. M. \& Hille, B. (1980). The permeability of end plate channels to monovalent and divalent metal cations. F. gen. Physiol. 75, 493-510.

Anderson, C. R. \& Stevens, C. F. (1973). Voltage clamp analysis of acetylcholine produced endplate current fluctuations at frog neuromuscular junction. F. Physiol., Lond. 235, 655-691.

AnHolt, R., Lindstrom, J. \& Montal, M. (1980). Functional equivalence of monomeric and dimeric forms of purified acetylcholine receptors from Torpedo californica in reconstituted lipid vesicles. Eur. F. Biochem. 109, $48 \mathrm{I}-487$.

Antanavage, J., Chien, T. F., Ching, Y. C., Dunlap, L., Mueller, P. \& RUDY, B. (1978). Formation and properties of cell-size single bilayer vesicles. Biophys. F. 21, $122 a$.

Antanavage, J., Chien, T. F., Ching, Y. C., Dunlop, C. \& Mueller, P. (1977). Rhodopsin mediated proton fluxes in lipid bilayers. Biophys. F. r7, I82a.

A.pplebury, M. L., Zuckerman, D. M., Lamola, A. A. \& Jovin, T. M. (1974). Rhodopsin purification and recombination with phospholipids assayed by the metarhodopsin I-metarhodopsin II transition. Biochemistry N.Y. 13, 3448-58.

Bamberg, E., Apell, H. J., Dencher, N., Sperling, W., Stieve, H. \& LÄUGER, P. (1979). Photocurrents generated by bacteriorhodopsin in planar bilayer membranes. Biophys. Struct. Mechanism 5, 277-292. 
Barrell, B. G., Bankier, A. T. \& Dvouin, J. T. (1979). A different genetic code in human mitochondria. Nature, Lond. 282, 189-194.

BAYLOR, D. A., Hodgkin, A. L. \& LAMB, T. L. (1974). Reconstruction of the electrical responses of turtle cones to flashes and steps of light. 7 . Physiol., Lond. 242, 759-791.

BAYLOR, D. A., LAMB, T. D. \& YAU, K.-W. (I979). Responses of retinal rods to single photons. F. Physiol., Lond. 288, 613-634.

Benz, R., Janko, K., Boos, W. \& LäUger, P. (1978). Formation of large, ion-permeable membrane channels by the matrix protein (porin) of Escherichia coli. Biochim. biophys. Acta 511, 305-319.

Benz, R., JANKo, K. \& LÄUGER, P. (I979). Ionic selectivity of pores formed by the matrix protein (porin) of Escherichia coli. Biochim. biophys. Acta 55I, 238-247.

Bitensky, M. W., Wheeler, G. L., Aloni, B., Vetury, S. \& Matuo, Y. (1978). Light- and GTP-activated photoreceptor PDE regulation by a light-activated GTPase and identification of rhodopsin as the binding site. Adv. Cyclic Nucleotide Res. 9, 553-572.

Bogomolni, R. A., Baker, R. A., Lozier, R. H. \& Stoeckenius, W. (1976). Light-driven proton translocations in Halobacterium halobium. Biochim. biophys. Acta 440, 68-88.

Bogomolni, R. A., Baker, R. A., Lozier, R. H. \& Stoeckenius, W. (1980). Action spectrum and quantum efficiency for proton pumping in Halobacterium halobium. Biochemistry, N.Y. 19, $2151-2159$.

Briley, M. S. \& ChangeUX, J. P. (1977). Isolation and purification of the nicotinic acetylcholine receptor and its functional reconstitution into a membrane environment. Int. Rev. Neurobiol. 20, 3 I-63.

CASEY, R. P., Chappell, J. B. \& Azzi, A. (1979). Limited-turnover studies on proton translocation in reconstituted cytochrome $c$ oxidase-containing vesicles. Biochem. F. 182, I49-156.

CASEY, R. P., Thelen, M. \& AzzI, A. (I980). Dicyclohexylcarbodiimide binds specifically and covalently to cytochrome $c$ oxidase while inhibiting its $\mathrm{H}^{+}$-translocating activity. F. biol. Chem. 255, 3994-4000.

Cattel, K. J., Knight, I. G., Lindop, C. R. \& BeEchey, R. B. (1970). The isolation of dicyclohexylcarbodiimide-binding proteins from mitochondrial membranes. Biochem. $\mathcal{F}$. 1r7, 1011-ror3.

Chang, H. W. \& Bock, E. (1977). Molecular forms of acetylcholine receptor. Effect of calcium ions and a sulfhydryl reagent on the occurrence of oligomers. Biochemistry, N.Y. 16, 4513-4520.

Changeux, J.-P., Benedetti, E. L., Bourgeois, J.-P., Brisson, A., Cartaud, J., Devaux, P., Grunhagen, H., Moreau, M., Popot, J.-L., Sobel, A. \& WEBER, M. (1975). Some structural properties of cholinergic receptor protein in its membrane environment relevant to its function as a pharmacological receptor. Cold Spring Harbor Symp. quant. Biol. 40, 21 1-230.

Changeux, J.-P., Heidmann, T., Popot, J.-L. \& Sobel, A. (1979). Reconstitution of a functional acetycholine receptor under defined conditions. FEBS Lett. 105, 181-187. 
Chapron, Y. (1979). Observation d'un photopotentiel transitoire de la rhodopsine en préparation vesiculaire de membranes de bâtonnets rétiniens. C. r. hebd. Séanc. Acad. Sci., Paris 288, $155^{-1} 58$.

Chien, T. F. \& Mueller, P. (1976). The reconstitution of cytochrome oxidase proteolipid into bilayer membranes. Fedn Proc. Fedn Am. Socs. exp. Biol. 35, 1599.

Cohen, F. S., Zimmerberg, J. \& Finkelstein, A. (1980). Fusion of phospholipid vesicles with planar phospholipid bilayer membranes. II. Incorporation of a vesicular membrane marker into the planar membrane. $\mathcal{f}_{\text {. gen }}$ Physiol. 77, 251-270.

Colombini, M. (1979). A candidate for the permeability pathway of the outer mitochondrial membrane. Nature, Lond. 279, 643-645.

Colombini, M. (1980). Pore size and properties of channels from mitochondria isolated from Neurospora crassa. F. Membrane Biol. 53, 79-84.

CoNe, R. A. \& PAK, W. L. (1971). The early receptor potential. In Handbook of Sensory Physiology, vol. I (ed. W. R. Lowenstein), pp. 345-365. Berlin, Heidelberg \& New York: Springer-Verlag.

ConTr, F. \& NEHER, E. (1980). Single channel recordings of $\mathrm{K}^{+}$currents in squid axons. Nature, Lond. 285, 140-143.

Coronado, R. \& Milleer, C. (1979). Voltage-dependent Cs+ block of a $\mathrm{K}^{+}$ channel from sarcoplasmic reticulum. Nature, Lond. 280, 807-810.

Crofts, A. A., Crowther, D., Celis, H., De Celis, S. A. \& Tierney, G. (1977). Proton pumps in bacterial photosynthesis. Biochem. Soc. Trans. 5, 49I-495.

DanchaZY, Z. \& KaRVALY, B. (1976). Incorporation of bacteriorhodopsin into a bilayer lipid membrane: A photoelectric-spectroscopic study. FEBS Lett. 72, 136-138.

Danchazy, Z., Ormos, P., Drachev, L. A. \& Skulachev, V. P. (1978). Investigation by focussed laser beam scanning of the photoelectric activity of bacteriorhodopsin-containing lipid bilayers. Biophys. Y. 24, 423-428.

Darszon, A., Blair, L. \& Montal, M. (I979a). Purified rhodopsin-phosphatidylcholine complexes in hexane: Formation and characterization. FEBS Lett. 107, 213-216.

Darszon, A., Montal, M. \& Philipp, M. (i977a). Formation of detergentfree proteolipids from biological membranes: Application to rhodopsin. FEBS Lett. 14, 135.

Darszon, A., Montal, M. \& Zarco, J. (1977b). Light increases the ion and non-electrolyte permeability of rhodopsin-phospholipid vesicles. Biochem. biophys. Res. Commun. 76, 820-827.

Darszon, A., Philipp, M. Zarco, J. \& Montal, M. (1978). Rhodopsinphospholipid complexes in apolar solvents: Formation and properties. f. Membrane Biol. 43, 71-9o.

Darszon, A., Strasser, R. J.\& Montal, M. (1979b). Rhodopsin phospholipid complexes in apolar environments: Photochemical characterization. Biochemistry, N.Y. 18, 5205-5213. 
Darszon, A., Vandenberg, C. A., Ellisman, M. H. \& Montal, M. (1979c). Incorporation of membrane proteins into large single bilayer vesicles: Application to rhodopsin. F. Cell. Biol. 81, 446-452.

Darszon, A., Vandenberg, C. A., Schönfeld, M., Ellisman, M. H., Spitzer, N. \& Montal, M. (1980). Reassembly of protein-lipid complexes into large bilayer vesicles: Perspectives for membrane reconstitution. Proc. natn. Acad. Sci. U.S.A. 77, 239-243.

DAS, M. L. \& CRANE, F. L. (1964). Proteolipids. I. Formation of phospholipid cytochrome $c$ complexes. Biochemistry, N.Y. 3, 696-700.

Devillers-Thiery, A., Changeux, J.-P., Paroutaud, P. \& Strosberg, A. D. (1979). The amino-terminal sequence of the 40000 molecular weight subunit of the acetylcholine receptor protein from Torpedo marmorata. FEBS Lett. 104, 99-105.

Dionne, V. E., Steinbach, J. H. \& Stevens, C. F. (1978). Voltage dependence of agonist effectiveness at the frog neuromuscular junction. J. Physiol. 281, 4 21-444.

Drachev, L. A., Frolov, V. M., Kaulen, A. D., Kondrashin, A. A., Samuilov, V. D., Semenov, A. Yu. \& Skulachev, V. P. (1976a). Generation of electric current by chromatophores of Rhodospirillum Rubrum and reconstitution of electrogenic function in subchromatophore pigment-protein complexes. Biochim. biophys. Acta. 440, 637660.

Drachev, L. A., Frolov, V. M., Kaulen, A. D., Lieberman, E. A., Ostroumov, S. A., Plakunova, V. G., Semenov, A. Yu. \& Skulachev, V. P. $(1976 b)$. Reconstitution of biological molecular generators of electric current: Bacteriorhodopsin. F. Biol. Chem. 251, 70597065 .

Drachev, L. A., Kaulen, A. D. \& Skulachev, V. P. (1978). Time resolution of the intermediate steps in the bacteriorhodopsin-linked electrogenesis. FEBS Lett. 87, 161-167.

Drachev, L. A., Kondrashin, A. A., Samuilov, V. V. \& Skulachev, V. P. (1975). Generation of electric potential by reaction centre complexes from Rhodospirillum Rubrum. FEBS Lett. 50, 219-222.

Drachev, L. A., Jasaitis, A. A., Koulen, A. D., Kondrashin, H. A., Liberman, E. A., Hayrecek, I. B., Ostroumov, S. A., Semenov, A. Y. \& Skulachev, V. P. (1974). Direct measurement of electric current generation by cytochrome oxidase, $\mathrm{H}^{+}$-ATPase and bacteriorhodopsin. Nature, Lond. 249, 321-323.

Dwyer, T. M., Adams, D. J. \& Hille, B. (1980). The Permeability of the end plate channel to organic cations in frog muscle. $\mathcal{F}$. gen. Physiol. 75, 469-492.

EPSTEIN, M. \& RACKER, E. (1978). Reconstitution of carbamylcholinedependent sodium ion flux and desensitization of the acetylcholine receptor from Torpedo californica. f. biol. Chem. 253, 6660-6662.

Fambrough, D. (1979). Control of acetylcholine receptors in skeletal muscle. Physiol. Rev. 59, 165-227. 
Fener, G. \& Okamura, M. Y. (1978). Chemical composition and properties of reaction centres. In The Photosynthetic Bacteria (ed. R. K. Clayton and W. R. Sistrom), pp. 349-386.

Fesenko, E. E. \& LyvbaRsky, A. (1977). Effects of light on artificial lipid membranes modified by photoreceptor membrane fragments. Nature, Lond. 268, 562-563.

Fesenko, E. E., Ratner, V. L. \& Lyvbarsky, A. (1976). The study of photoconduction of artificial lipid membranes incorporating rhodopsin. The simultaneous changes of membrane conduction and rhodopsin fluorescence. Mol. Biol. Rep. 3, 175-1 79 .

Fillingame, R. H. (1976). Purification of the carbodiimide-reactive protein component of the ATP energy-transducing system of Escherichia coli. f. biol. Chem. 251, 6630-6637.

Fuller, S. D., Capaldi, R. A. \& Henderson, R. (I979). Structure of cytochrome $c$ oxidase in deoxycholate-derived two-dimensional crystals. f. molec. Biol. 134, 305-327.

Fung, B. K.-K. \& Stryer, L. (I980). Photolyzed rhodopsin catalyzes the exchange of GTP for bound GDP in retinal rod outer segments. Proc. natn. Acad. Sci. U.S.A. 77, 2500-2504.

GAGE, P. (1976). Generation of end-plate potentials. Physiol. Rev. 56, 177247.

Garavito, R. M. \& Rosenbusch, J. P. (1980). Three-dimensional crystals of an integral membrane protein: An initial X-ray analysis. f. Cell Biol. 86, 327-329.

Gemant, A. (1962). Ions in hydrocarbons, p. 26r. New York: Wiley.

GitleR, C. \& MonTal, M. (1972a). Thin-proteolipid films: A new approach to the reconstitution of biological membranes. Biochem. biophys. Res. Commun. 47, 1486-1491.

GitleR, C. \& Montal, M. (I972b). Formation of decane-soluble proteolipids: Influence of monovalent and divalent cations. FEBS Lett. 28 , 329-332.

Gold, G. H. \& KoRENBrot, J. J. (1980). Light-induced calcium release by intact retinal rods. Proc. natn. Acad. Sci. U.S.A. 77, 5557-556r.

Gonsalez-Ross, J. M., Paraschos, A. \& Martinez-Carrion, M. (ig80). Reconstitution of functional membrane-bound acetylcholine receptor from isolated Torpedo californica receptor protein and electroplax lipids. Proc. natn. Acad. Sci. U.S.A. 77, 1796-1800.

Greengard, P. (1978). Phosphorylated proteins as physiological effectors. Science 199, $146-152$.

Hagins, W. A. (1972). The visual process; Excitatory mechanisms in the primary receptor cells. A. Rev. Biophys. Bioeng. 1, 131-158.

Hagins, W. A. \& Yoshikami, S. (1977). Intracellular transmission of visual excitation in photoreceptors: electrical effects of chelating agents introduced into rods by vesicle fusion. In Vertebrate Photoreception (ed. H. B. Barlow and P. Fatt), pp. 97-139. New York: N.Y. Academic Press. 
Hamilton, S. L., Mclaughlin, M. \& Karlin, A. (1979). Formation of disulfide-linked oligomers of acetylcholine receptor in membranes from Torpedo electric tissue. Biochemistry, N.Y. 18, I 55-163.

Hancock, R. E. W., Decad, G. M. \& Nikaido, H. (I979). Identification of the protein producing trans-membrane diffusion pores in the outer membrane of Pseudumonas Aeruginosa PAOI. Biochim. biophys. Acta $554,323-331$.

Hartig, P. R. \& Raftery, M. A. (1979). Preparation of right side-out acetylcholine receptor enriched intact vesicles from Torpedo californin electroplaque membranes. Biochemistry, N.Y. 18, $1146-1156$.

HeidmanN, T. \& ChangeuX, J.-P. (1978). Structural and functional properties of the acetylcholine receptor protein in its purified and membranebound states. A. Rev. Biochem. 47, 317-357.

Henderson, R. (1977). The purple membrane from Halobacterium halobium. A. Rev. Biophys. Bioengineer 6, 87-ıog.

Henderson, R., Capaldi, R. A. \& Leigh, J. S. (1977). Arrangement of cytochrome oxidase moelcules in two-dimensional vesicles crystals. f. molec. Biol. 112, $631-648$.

HerrmanN, T. R. \& Rayfield, G. W. (1978). The electrical response to light of bacteriorhodopsin in planar membranes. Biophys. F. 21, I I 1-125.

Hess, G. P., Cash, D. J. \& Aoshima, H. (1979). Acetylcholine receptorcontrolled ion fluxes in membrane vesicles investigated by fast reaction techniques. Nature, Lond. 282, 329-331.

Hinkle, P. C. (1973). Electron transfer across membranes and energy coupling. Fedn Proc. Fedn Am. Socs exp. Biol. 32, 1988-1992.

Hinkle, P. C., Kim, J. J. \& RACKer, E. (1972). Ion transport and respiratory control in vesicles formed from cytochrome oxidase and phospholipids. 7. biol. Chem. 247, $1338-1339$.

Hoffman, W., Siebert, F., Hofmann, K. P. \& Kreutz, W. (1978). Two distinct rhodopsin molecules within the disc membrane of vertebrate rod outer segments. Biochim. biophys. Acta 503, 450-461.

HoNG, K. \& Hubbell, W. L. (1973). Lipid requirements for rhodopsin regenerability. Biochemistry, N.Y. 12, 4517-4523.

Hong, F. T. \& MonTaL, M. (1979). Bacteriorhodopsin in model membranes: A new component of the displacement photocurrent in the microsecond time scale. Biophys. F. 25, 465-472.

HubBard, R. \& Wald, G. (1952). Cis-trans isomers of vitamin A and retinene in the rhodopsin system. F. gen. Physiol. 36, 269-315.

HubBell, W. L. \& Bownds, M. D. (1979). Visual transduction in vertebrate photoreceptors. A. Rev. Neurosci. 2, 17-34.

Hubbell, W. L., Fung, B. K.-K., Chen, Y. S. \& Hong, K. (1977). Molecular anatomy and light dependent processes in photoreceptor membranes. In Vertebrate Photoreception (ed. H. B. Barlow and P. Fatt), pp. 4I-59. New York: N.Y. Academic Press.

Huganir, R. L., Shell, M. A. \& Racker, E. (1979). Reconstitution of the purified acetylcholine from Torpedo californica. FEBS Lett. 108, 155-160. 
Hwang, S.-B., Korenbrot, J. I. \& Stoeckenius, W. (1977a). Structural and spectroscopic characteristics of bacteriorhodopsin in air-water interface films. $\mathcal{F}$. Membrane Biol. 36, I $15^{-1} 3^{6}$.

Hwang, S.-B., Korenbrot, J. I. \& Stoeckenius, W. (1977b). Proton transport by bacteriorhodopsin through an interface film. F. Membrane Biol. $36,137^{-1} 58$.

Hwang, S.-B., Korenbrot, J. I. \& Stoeckenius, W. (1978). Transient photovoltages in purple membrane multilayers: Charge displacements in bacteriorhodopsin and its photointermediates. Biochim. biophys. Acta 509, 300-308.

Jasaitis, A. A., Nemecek, I. B., Severina, I. I., Skulachev, V. P. \& Smirnova, S. M. (1972). Membrane potential generation by two reconstituted mitochondrial systems: Liposomes inlayed with cytochrome oxidase or with ATPase. Biochim. biophys. Acta 275, 485-490.

Jenkinson, D. H. (1960). The antagonism between tubocurarine and substances which depolarize the motor end-plate. $\mathcal{f}$. Physiol. 152, 309-324.

Kagawa, Y., Sone, N., Hirata, H. \& Yoshida, M. (1979). Structure and function of $\mathrm{H}^{+}$-ATP-ase. 7 . Bioenerg. Biomembr. Ir, 39-78.

KARLIN, A. (1980). Molecular properties of nicotinic acetylcholine receptors. In Cell Surface Reviews, vol. 6 (ed. G. Poste, G. Nicholson and C. N. Cotman). New York: Elsevier-North Holland. Vol. vi, pp. I9I-260.

Karlin, A., Weill, C. L., McNamee, M. G. \& Valderrama, R. (1975). Facets of the structure of acetylcholine receptors from Electrophorus and Torpedo. Cold Spring Harb. Symp. quant. Biol. 40, 203-213.

Kasahara, M. \& HinkLE, P. C. (1976). Reconstitution of D-glucose transport catalyzed by a protein faction from human erythrocytes in sonicated liposomes. Proc. natn. Acad. Sci. U.S.A. 73, 396-400.

Kasai, M. \& ChangeuX, J.-P. (197I). In vitro excitation of purified membrane fragments by cholinergic agonists. $\mathcal{F}$. Membrane Biol. 6, I-80.

Katz, B. \& Miledi, R. (1972). The statistical nature of the acetylcholine potential and its molecular components. F. Physiol. 224, 665-699.

Katz, B. \& TheslefF, S. (1957). A study of the desensitization produced by acetylcholine at the motor endplate. F. Physiol. 138, 63-80.

Kendall-Tobias, M. \& CrofTs, A. R. (1979). Reaction centres in hexane. Biophys. F. 25, 54a.

KeszTHELYI, L. \& ORMOS, P. (1980). Electric signals associated with the photocyle of bacteriorhodopsin. FEBS Lett. 109, I89-193.

Kilian, P. L., Dunlap, C. R., Mueller, P., Schell, M. A., Huganir, R. L. \& RACKER, E. (I980). Reconstitution of acetylcholine receptor from Torpedo californica with highly purified phospholipids: effect of $\alpha$ tocopherol, phylloquinone, and other terpenoid quinones. Biochem. biophys. Res. Commun. 93, 409-414.

Klymkowsky, M. W., Heuser, J. E. \& Stroud, R. M. (1980). Protease effects on the structure of acetylcholine receptor membranes from Torpedo californica. F. Cell Biol. 85, 823-838. 
KORENBROT, J. I. (1977). Ion transport in membranes: Incorporation of biological ion translocating proteins in model membrane systems. A. Rev. Physiol. 39, 19-49.

Korenbrot, J. I. \& Hwang, S.-B. (1980). Proton transport by bacteriorhodopsin in planar membranes assembled from air-water interface films. F. gen. Physiol. 76, 649-682.

Korenbrot, J. I. \& PrAmiK, M. J. (1977). Formation, structure, and spectrophotometry of air-water interface films containing rhodopsin. $\mathcal{F}$. Membrane Biol. 37, 235-262.

KRAB, K. \& WiKstRÖM, M. (1978). Proton-translocating cytochrome $c$ oxidase in artificial phospholipid vesicles. Biochim. biophys. Acta 504, 200-214.

Labarca, P., Coronado, R. \& Miller, C. (i980). Thermodynamic and kinetic studies of the gating behavior of a $\mathrm{K}^{+}$-selective channel from the sarcoplasmic reticulum membrane. F. gen. Physiol. 76, 397-424.

Latorre, R. \& Alvarez, O. (1980). Voltage-dependent channels in planar lipid bilayers. Physiol. Rev. (in the Press).

Lees, M. B., Sakura, J. D., Sapirstein, V. S. \& Curatolo, W. (1979). Structure and function of proteolipids in myelin and non-myelin membranes. Biochim. biophys. Acta 559, 209-230.

Lemberg, M. R. (1969). Cytochrome oxidase. Physiol. Rev. 49, 48-121.

Liebman, P. A. \& PUGH JR., E. N. (I979). The control of phosphodiesterase in rod disk membranes: kinetics, possible mechanism and significance for vision. Vision Res. 19, 375-380.

Lindstrom, J., Anholt, R., Einarson, B., Engel, A., Osame, M. \& Montal, M. (1980a). Purification of acetylcholine receptors, reconstitution into lipid vesicles, and study of agonist-induced cation channel regulation. f. biol. Chem. 255, 8340-8350.

Lindstrom, J., Gullick, W., Conti-Tronconi, B. \& Ellisman, M. (ig8o b). Proteolytic nicking of the acetylcholine receptor. Biochemistry, N.Y. I9, 479I-4795.

Lindstrom, J., Merlie, J. \& Yogeeswaran, G. (1979). Biochemical properties of acetylcholine receptor subunits from Torpedo, Californica. Biochemistry, N.Y. 18, 4465-4470.

Lindstrom, J. \& Patrick, J. (I974). Purification of acetylcholine receptors by affinity chromatography. In Synaptic Transmission and Neuronal Interaction (ed. M. V. L. Bennett), pp. I9I-2 I6. New York: Raven Press.

Lipton, S. A., Rasmussen, H. \& Dowling, J. E. (1977). Electrical and adaptive properties of rod photoreceptors in Bufo marinus. II. Effects of cyclic nucleotides and prostaglandins. F. gen. Physiol. 70, 77I-791.

Lozier, R. H., Bogomolni, R. A. \& Stoeckenius, W. (1975). Bacteriorhodopsin: A light driven proton pump in Halobacterium halobium. Biophys. F. 15, 955-962.

Ludwig, B. \& Schatz, G. (1980). A two-subunit cytochrome $c$ oxidase (cytochrome A, $\mathrm{A}_{3}$ ) from Paracoccus dentrificans. Proc. natn. Acad. Sci. U.S.A. 77, 196-200. 
Maclennan, D. F. \& Holland, P. C. (1975). Calcium transport in sarcoplasmic reticulum. A. Rev. Biophys. Bioeng. 4, 377-404.

Magleby, K. L. \& Stevens, C. F. (1972). A quantitative description of endplate currents. F. Physiol. Lond. 223, I73-197.

Michel, H. \& Oesterhelt, D. (1980). Three-dimensional crystals of membrane protein: Bacteriorhodopsin. Proc. natn. Acad. Sci. U.S.A. 77, $1283-1285$.

Miki, N., Baraban, J. M., Keirns, J. J., Boyce, J. J. \& Bitensky, M. W. (1975). Purification and properties of the light-activated cyclic nucleotide phosphodiesterase of rod outer segments. F. biol. Chem. 250, 63206327 .

MILlER, C. (1978). Voltage-gated cation conductance channel from fragmented sarcoplasmic reticulum: Steady-state electrical properties. 7. Membrane Biol. 40, I-23.

Miller, C., Arvan, P., Telford, J. N. \& Racker, E. (ig76). Calciuminduced fusion of proteoliposomes: Dependence on transmembrane osmotic gradient. $\mathcal{F}$. Membrane Biol. 30, $27 \mathrm{r}-282$.

MilleR, C. \& RACKER, E. (1976). $\mathrm{Ca}^{2+}$-induced fusion of fragmented sarcoplasmic reticulum with artificial bilayers. F. Membrane Biol. 30, 283-300.

Miller, W. H. \& Nicol, G. D. (I979). Evidence that cyclic GMP regulates membrane potential in rod photoreceptors. Nature, Lond. 280, 6466.

Mitchell, P. (1968). Chemiosmotic Coupling and Energy Transduction Glynn Research, Bodmin, United Kingdom.

MonTAL, M. (1974). Lipid-protein assembly and the reconstitution of biological membranes. In Perspectives in Membrane Biology (ed. S. Estrada-O. and C. Gitler), pp. 59I-522. New York: Academic Press.

Montal, M. (1975). Rhodopsin in experimental membranes: An approach to elucidate its role in the process of phototransduction. In Molecular Aspects of Membrane Phenomena (ed. H. R. Kaback, G. K. Radda, R. Schwyzer, H. Neurath and W. R. Wiley), pp. 316-338. New York, Berlin, Heidelberg: Springer-Verlag.

Montal, M. (1976). Experimental membranes and mechanisms of bioenergy transduction. A. Rev. Biophys. Bioeng. 5, I19-175.

Montal, M. (1979). Rhodopsin in model membranes. Biochim. biophys. Acta 559, 23 I-257.

Montal, M., Darszon, A., Korenbrot, J. I. \& Trissl, H. W. (1975). Light induced effects in lipid bilayers with incorporated Rhodopsin. Vth Int. Biophys. Congr., Copenhagen, Denmark, August, 1975. Abstract, p. 177.

Montal, M., Darszon, A. \& Strasser, R. J. (1978). Rhodopsin and bacteriorhodopsin in model membranes. In Frontiers in Biological Energetics, vol. 2 (ed. P. L. Dutton, J. Leigh and A. Scarpa), pp. Iro9-IIr8. New York: Academic Press.

Montal, M., Darszon, A. \& Trissl, H. W. (I977). Transmembrane channel formation in rhodopsin-containing bilayer membranes. Nature, Lond. 267, $221-225$. 
Montal, M. \& KorenBrot, J. I. (I973). Incorporation of rhodopsin proteolipid into bilayer membranes. Nature, Lond. 246, 219-22 I.

Montal, M. \& Mueller, P. (1972). Formation of bimolecular membranes from lipid monolayers and a study of their electrical properties. Proc. natn Acad. Sci. U.S.A. 69, 356 $\mathrm{I}-3566$.

Mueller, P., Rudin, D. O., Tien, H. Tr. \& Wescott, W. C. (1962). Reconstitution of cell membrane structure in vitro and its transformation into an excitable system. Nature, Lond. 194, 979-981.

NaKaE, T. $(1976 a)$. Identification of the outer membrane protein of $E$. coli that produces transmembrane channels in reconstituted vesicle membranes. Biochem. biophys. Res. Commun. 71, 877-889.

NAKAE, T. $(1976 b)$. Outer membrane of Salmonella. Isolation of protein complex that produces transmembrane channels. f. biol. Chem. 25I, 2176-2178.

Nathanson, N. M. \& Hall, Z. W. (1979). Subunit structure and peptide mapping of junctional and extrajunctional acetylcholine receptors from rat muscle. Biochemistry, N.Y. 18, 3392-340I.

Negrin, R. S., Foster, D. L. \& Fillingame, R. H. (1980). Energy-transducing $\mathrm{H}^{+}$-ATP-ase of Escherichia coli. Reconstitution of proton translocation activity of the intrinsic membrane sector. 7 . biol. Chem. $255,5643-5648$.

Neher, E, \& Sakmann, B. (1976). Single channel currents recorded from membrane of denervated frog muscle fibres. Nature, Lond. 260, 799-802.

Neher, E. \& Steinbach, J. H. (1978). Local anaesthetics transiently block currents through single acetylcholine receptor channels. F. Physiol. 277, $153-176$.

Nelson, N., Anholt, R., Lindstrom, J. \& Montal, M. (I980). Reconstitution of purified acetylcholine receptors with functional ion channels in planar lipid bilayers. Proc. natn Acad. Sci. U.S.A. 77, 3057-306r.

Nelson, N., Eytan, E., Notsani, B. E., Sigrist, H., Sigrist-Nelson, K. \& Gitler, C. (1977). Isolation of a chloroplast $N, N^{\prime}$-dicyclohexylcarbodiimide-binding proteolipid active in proton translocation. Proc. natn. Acad. Sci. U.S.A. 24, 2375-2378.

Neubig, G. R. \& Cohen, J. B. (1980). Permeability control of cholinergic receptors in Torpedo post synaptic membranes: Agonist dose-response relations measured at second and millisecond times. Biochemistry, N.Y. I9, 2770-2779.

Neubig, R., Krodel, E. K., Boyd, N. D. \& Cohen, J. B. (1979). Acetylcholine and local anesthetic binding to Torpedo nicotinic postsynaptic membranes after removal of nonreceptor peptides. Proc. natn. Acad. Sci. U.S.A. 76, 690-694.

NikaIDo, H. \& NAKaE, T. (1979). The outer membrane of Gram-negative bacteria. Adv. Microb. Physiol. 20, 163-250.

O'Brien, D. F., Costa, L. F. \& OTT, R. A. (I977a). Photochemical functionality of rhodopsin-phospholipid recombinant membranes. Biochemistry, N.Y. 16, 1295-1303. 
O'Brien, D. F., Zambulyadis, N., Michaels, F. M. \& Ott, R. (1977b). Light-regulated permeability of rhodopsin: egg phosphatidylcholine recombinant membranes. Proc. natn. Acad. Sci. U.S.A. 74, 52225226.

Okamoto, H., Sone, N., Hirata, H., Yoshida, M. \& Kagawa, Y. (1977). Purified proton conductor in proton translocating adenosine triphosphatase of a thermophyllic bacterium. $\mathcal{F}$. biol. Chem. 252, 6125-6131.

Ostroy, S. E. (1977). Rhodopsin and the visual process. Biochim. biophys. Acta 463, 9I-175.

Ovchinnikov, Yu. A., Abdulaev, N. G., Feigina, M. Yu., Kiselev, A. V. \& Lobanov, N. Na. (1979). The structural basis of the functioning of bacteriorhodopsin: An overview. FEBS. Lett. roo, $219-224$.

Ozawa, T., Suzuki, H. \& Tanaka, M. (1980). Crystallization of part of the mitochondrial electron-transfer chain: cytochrome $c$ oxidase-cytochrome $c$ complex. Proc. natn Acad. Sci. U.S.A. 77, 928-930.

Packham, N. K., Packham, C., Mueller, P., Tiede, D. M. \& Dutton, P. L. (1980). Reconstitution of photochemically active reaction centres in planar phospholipid membranes. FEBS Lett. 1 10, Ior-106.

Papermaster, D. S. \& DreYer, W. J. (1974). Rhodopsin content in outer segment membranes of bovine and frog retinal rods. Biochemistry, N.Y. 13, 2438-2444.

Patlak, J., Gration, K. A. F. \& Usherwood, P. N. R. (1979). Single glutamate channels in locust muscle. Nature, Lond. 278, 643-645.

Pattus, F. Desnuelle, P. \& Verger, R. (1978a). Spreading of liposomes at the air-water interface. Biochim. biophys. Acta 507, 62-70.

Patrus, F., Piorant, M. C. L., Lardunski, C. J., Desnuelle, P. \& Verger, R. ( $1978 b)$. Spreading of biomembranes at the air-water interface. Biochim. biophys. Acta 507, 71-82.

PenN, R. D. \& HaGins, W. A. (1972). Kinetics of photocurrent of retinal rods. Biophys. F. 12, 1073-1094.

Polans, A. S., Hermolin, J. \& Bownds, D. (1979). Light induced dephosphorylation of frog rod outer segment proteins. F. gen. Physiol. 74, $595-6 \times 3$.

Popot, J. L., Demel, R. A., Sobel, A., van Deenen, L. L. M. \& Changeux, J.-P. (1978). Interaction of the acetylcholine (nicotinic) receptor protein from Torpedo marmorata electric organ with monolayers of pure lipids. Eur. F. Biochem. 85, 27-42.

Prochaska, L., Bisson, R. \& Capaldi, R. A. (1980). Structure of cytochrome $c$ oxidase complex: Labeling by hydrophilic and hydrophobic protein modifying reagents. Biochemistry, N.Y. 19, 3174-3179.

RACKER, E. (1973). A new procedure for the reconstitution of biological active phosopholipid vesicles. Biochem. biophys. Res. Commun. 55, 224230.

Racker, E. \& Stoeckenius, W. (I974). Reconstitution of purple membrane vesicles catalyzing light-driven proton uptake and adenosine triphosphate formation. f. biol. Chem. 249, 662-663. 
Raftery, M. A., Hunkapiller, M. W., Strader, C. D. \& Hood, L. A. (ig80). Acetylcholine receptor: complex of homologous subunits. Science, N.Y. 208, $1454-1457$.

Raftery, M. A., Vandlen, R. L., Reed, K. L. \& Lee, T. (1975). Structure and functional characteristics of acetylcholine receptor. Cold. Spring Harb. Symp. quant. Biol. 40, 193-202.

Ramakrishnan, V., Darszon, A., Philipp, M. \& Montal, M. (i980). Rhodopsin in model membranes: The kinetics of channel opening and closing in rhodopsin-containing planar lipid bilayers. Ann. N.Y. Acad. Sci. 358, 36-42.

Rang, H. P. (1975). Acetylcholine receptors. Q. Rev. Biophys. 7, 283399.

REYNOLDS, J. \& KARLIN, A. (1978). Molecular weight in detergent solution of acetylcholine receptor from Torpedo californica. Biochemistry, N.Y. 17, $2035^{-2038}$.

Robinson, W. E. \& Hagins, W. A. (1977). A light activated GTPase in retinal rod outer sements. Biophys. F. 17, $196 a$.

RosensusCh, J. P. (1974). Characterization of the major envelope protein from Escherichia coli. F. biol. Chem. 249, 8019-8029.

Rosenfeld, T., Honig, B., Ottolengli, M., Hurley, J. \& Ebrey, T. F. (1977). Cis-trans isomerization in the photochemistry of vision. Pure appl. Chem. 49, 34I-35I.

Sakmann, B., Patlak, J. \& Neher, E. (1980). Single acetylcholine-activated channels show burst-kinetics in presence of desensitizing concentrations of agonist. Nature, Lond. 286, 7 I-73.

Salem, L. (1962). The role of long-range forces in the cohesion of lipoproteins. Can. F. Biochem. Physiol. 40, 1287-1298.

Schein, S. J., Colombini, M. \& Finkelstein, A. (1976). Reconstitution in planar lipid bilayers of a voltage-dependent anion-selective channel obtained from Paramecium mitochondria. 7. Membrane Biol. 30, 99I 20.

SCHERRER, R. \& GERHARD'T, P. (197I). Molecular sieving by bacillus megaterium cell wall and protoplast. $\mathcal{f}$. Bact. 107, 7 18-728.

SCHINDler, H. (I979a). Autocatalytic transport of the peptide antibiotics suzukacillin and alamethicin across lipid membranes. FEBS Lett. ro4, I 57 - I60.

Schindler, H. (1979b). Exchange and interactions between lipid layers at the surface of a liposome solution. Biochim. biophys. Acta 555, 316336 .

SchiNDLER, H. (I $800 a$ ). Formation of planar bilayers from artificial or native membrane vesicles. FEBS Lett. 122, 77-79.

ScHINDLER, H. ( $1980 b)$. Methods for reconstituting receptors in planar membranes. NRP Bulletin. (In the Press).

SchindLER, H. \& QUAST, U. (1980). Functional acetylcholine receptor from Torpedo marmorata in planar membranes. Proc. natn. Acad. Sci. U.S.A. 77, 3052-3056. 
Schindler, H. \& Rosenbusch, J. (1978). Matrix protein from Escherichia coli outer membranes forms voltage controlled channels in lipid bilayers. Proc. natn. Acad. Sci. U.S.A. 75, 3751-3755.

SCHINDleR, H. \& RosenbusCh, J. P. (I98I). Matrix protein in planar membranes: Clusters of channels in a native environment and their functional reassembly. Proc. natn. Acad. Sci. U.S.A. (In the Press.)

Schindler, H., Rosendusch, J. P. \& Quast, U. (ig8o). A novel concept of membrane reconstitution applied to acetylcholine receptor from Torpedo and matrix protein from Escherichia coli. Neurochem. Intl. 2, 29I298.

SchnetKamp, P. P. M. (1980). Ion selectivity of the cation transport system of isolated intact cattle rod outer segments. Evidence for a direct communication between the rod plasma membrane and the rod disk membrane. Biochim. biophys. Acta. 598, 66-90.

Schönfeld, M., Montal, M. \& Feher, G. (1979). Functional reconstitution of photosynthetic reaction centres in planar lipid bilayers. Proc. natn Acad. Sci U.S.A. 76, 635 I-6355.

Schönfeld, M., Montal, M. \& FeHER, G. (1980). Reaction center - phospholipid complex in organic solvents; formation and properties. Biochemistry, N.Y. 19, I $535^{-1} 54^{2}$.

Sebald, W., Graf, T. \& Lukins, H. H. (1979). The dicyclohexylcarbodiimidebinding of the mitochondrial ATPase complex from Neurospora crassa and Saccharomyces cerevisiae. Identification and isolation. Eur. $\mathcal{F}$. Biochem. 93, 587-599.

Sebald, W., Machleid'T, W. \& Wachter, E. (I980). $N, N^{\prime}$-Dicyclohexylcarboldyimide binds specifically to a single glutamyl residue of the proteolipid subunit of the mitochondrial adenosinetriphosphatase from Neurospora crassa and Saccharomyces cerevisiae. Proc. natn Acad. Sci. U.S.A. 77, $785-789$.

Sebald, W. \& WAChTER, E. (1978). In Energy Conservation in Biological Membranes (ed. G. Schafer and M. Klingenberg), pp. 159-194, New York: Springer.

Shamoo, A. E. \& Murphy, T. J. (1979). Ionophores and ion transport across natural membranes. Curr. Tops Bioenerg. 9, 147-177.

Shinozawa, T., Uchida, S., Martin, E., Cafiso, D., Hubbell, W. L. \& Bitensky, M. W. (I980). Additional component required for activity and reconstitution of light activated vertebrate photoreceptor GTPase. Proc. natn. Acad. Sci. U.S.A. 77, 1408-1411.

Sigrist, H., Sigrist-Nelson, K. \& Gitrer, C. (1977). Single-phase butanol extraction: A new tool for proteolipid isolation. Biochem. biophys. Res. Commun. 74, 178-184.

SigwORTh, F. J. \& NeHER, E. (1980). Single $\mathrm{Na}^{+}$channel currents in cultured rat muscle cells. Nature, Lond. 287, 447-449.

Skulachev, V. P. (1975). Energy coupling in biological membranes; current state and perspectives. In Energy Transducing Mechanisms (ed. E. Racker), pp. 31-73. London, Baltimore: Butterworth. 
Sobel, A., Weber, M. \& Changeux, J.-P. (I977). Large scale purification of the acetylcholine-receptor protein in its membrane-bound and detergentextracted forms from Torpedo marmorata electric organ. Eur. F. Biochem. 80, $215-224$.

Sone, N., Yoshida, M., Hirata, H. \& Kagawa, Y. (1979). Resolution and reconstitution of proton translocating ATP-ase. In Cation flux across biomembranes, pp. 279-290. New York: Academic Press.

Stankowski, S. \& Gruenewald, B. (1980). Evaluation of co-operativity for phase transitions in two- and three-dimensional systems. Biophys. Chem. 12, $167-176$.

Steven, A. C., ten Heggeler, B., Müller, R., Kistler, J. \& Rosenbusch, J. P. (1977). Ultrastructure of a periodic protein layer in the outer membrane of Escherichia coli. F. Cell Biol. 72, 292-301.

Stoeckenius, W. (1980). Purple membrane of Halobacteria: A new light energy converter. Acc. Chem. Res. 13, 337-344.

Stoeckenius, W., Lozier, R. H. \& Bogomolni, R. A. (1979). Bacteriorhodopsin and the purple membrane of Halobacteria. Biochim. biophys. Acta 505, $215-278$.

TAKAGI, M. (1980). The electrical response of cephalopod visual cell membrane fragments. Photochem. \& Photobiol. 32, 539-545.

Takagi, M., Azuma, K. \& Kishimoto, V. (1965). A new method for the formation of bilayer membranes in aqueous solution. Annu. Rep. Biol. Works. Fac. Sci. Osaka Univ. r3, 107-1 10.

Thalenfold, B. \& Tzagoloff, A. (1980). Assembly of the mitochondrial membrane system. Sequence of the oxiz gene of yeast mitochondria DNA. F. biol. Chem. 255, 6173-6180.

TREDGold, R. H. \& Elgamal, M. (1979). A study of the incorporation of cytochrome oxidase into planar synthetic membranes. Biochim. biophys. Acta 555, 381-387.

TrișsL, H. W. (r979). Light-induced conformational changes in cattle rhodopsin as probed by measurements of the interface potential. Photochem. \& Photobiol. 29, 579-588.

Trissl, H. W., Darszon, A. \& MonTal, M. (1977). Rhodopsin in model membranes: Charge displacement in interfacial layers. Proc. natn. Acad. Sci. U.S.A. 74, 207-210.

Trissl, H. W. \& Montal, M. (r977). Electrical demonstration of rapid lightinduced conformational changes in bacteriorhodopsin. Nature, Lond. 266, 655-657.

Verger, R. \& PatTus, F. (1976). Spreading of membranes at the air-water interface. Chem. Phys. Lipids 16, 285-291.

Von Sengbusch, G. \& Srieve, H. (1971). Flash photolysis of rhodopsin. I. Measurements on bovine rod outer segments. Z. Naturf. B 26, 488-489.

Wald, G., Durrell, J. \& St George, R. C. C. (1950). The light reaction in the bleaching of rhodopsin. Science, N.Y. 11r, 179-18r.

Watanabe, S. \& Narahashi, T. (I979). Cation selectivity of acetylcholineactivated ionic channel of frog end plate. 7 . gen. Physiol. 74, 615-628. 
Weill, C. L., McNamee, M. G. \& Karlin, A. (1974). Affinity labelling of purified acetylcholine receptors from Torpedo californica. Biochem. biophys. Res. Commun. 6r, 997-1003.

Wheeler, G. L. \& Bitensky, M. W. (1977). A light-activated GTPase in vertebrate photoreceptors: regulation of light activated cyclic GMP phosphodiesterase. Proc. natn. Acad. Sci. U.S.A. 74, 3238-4242.

White, M. M. \& Miller, C. (1979). A voltage-gated anion channel from the electric organ of Torpedo californica. 7. biol. Chem. 254, 10161-10166.

Wikström, M. \& Krab, K. (1979). Proton-pumping cytochrome $c$ oxidase. Biochim. biophys. Acta, 549, 177-222.

Woodruff, M. L. \& Bownds, D. (1979). Amplitudes, kinetics and reversibility of light induced decrease in guanosine $3^{\prime}, 5^{\prime}$-cyclic monophosphate in isolated frog retinal rod outer segments. F. gen. Physiol. 73, 629-653.

Wu, W. C. S. \& Raftery, M. A. (1979). Carbamylcholine-induced rapid cation flux from reconstituted membrane vesicles containing purified acetylcholine receptor. Biochem. biophys. Res. Commun. 89, 26-35.

YEE, R. \& LIEBMAN, P. A. (1978). Light-activated phosphodiesterase of the rod outer segment. Kinetics and parameters of activation and deactivation. f. biol. Chem. 253, 8902-8909.

Yoshikami, S., George, J. S. \& Hagins, W. A. (I980). Light-induced calcium fluxes from outer segment layer of vertebrate retinas. Nature, Lond. 286, 395-398.

Yoshikami, S. \& Hagins, W. A. (1978). Calcium in excitation of vertebrate rods and cones; Retinal eflux of calcium studied with dichlorophosphonazo. III. Ann. N.Y. Acad. Sci. 307, 545-56I.

Yoshizawa, T. (1972). The behavior of visual pigments at low temperatures. In Handbook of Sensory Physiology, vol. VII/I (ed. H. J. A. Dartnall), pp. 146-179. Berlin, Heidelberg, New York: Springer.

ZAHLER, P. \& Niggli, V. (1977). The use of organic solvents in membrane research. In Methods in Membrane Biology, vol. 8 (ed. E. D. Korn), pp. I-50. New York and London: Plenum Press.

Zalman, L. S., Nikaido, H. \& Kagawa, Y. (i980). Mitochondrial outer membrane contains a protein-producing non-specific diffusion channels. f. biol. Chem. 255, 177 I-1 774 .

Zimmerberg, J., Cohen, F. S. \& Finkelstein, A. (1980). Fusion of phospholipid vesicles with planar phospholipid bilayer membranes. I. Discharge of vesicular contents across the planar membrane. 7 . gen. Physiol. 75, $24 \mathrm{I}-250$.

Zimmerman, W. F., Daemen, F. J. M. \& Bonting, S. J. (1976). Distribution of enzyme activities in subcellular fractions of bovine retina. $\mathcal{F}$. biol. Chem. 251, 4700-4707.

Zingsheim, H. P., Neugebauer, V.-Ch., Barrantes, F. J. \& Frank, J. (i 980). Structural details of membrane-bound acetylcholine receptor from Torpedo marmorata. Proc. natn. Acad. Sci. U.S.A. 77, 952-956. 